6 Abstract operation.

\title{
Stability criterion for the intensification of batch processes with model predictive control
}

\author{
Walter Kähm, Vassilios S. Vassiliadis* \\ Department of Chemical Engineering and Biotechnology, Process Systems Engineering Group, University of \\ Cambridge, West Cambridge Site, Philippa Fawcett Drive, CB3 OAS Cambridge, UK
}

Thermal runaways in batch processes can lead to significant issues for safety and performance during normal operation in industry. This is usually circumvented by running such processes at lower temperatures than necessary, hence losing the opportunity to intensify production and therefore reduce reaction time. The detection of the thermal stability of batch systems can potentially be embedded in an advanced control scheme, therefore improving the performance by being able to intensify the process, achieving higher yields while keeping a stable

The derivation of stability criterion $\mathcal{K}$ for high-order reactions is presented in this work, resulting in better control when embedded in Model Predictive Control (MPC) schemes than standard nonlinear MPC schemes, based on the work in Kähm and Vassiliadis (2018). The non-trivial extension of stability criterion $\mathcal{K}$ for multi-component reactions with application to MPC systems is discussed in detail. The logic and verification of the form of the resultant Damköhler number in particular is discussed and demonstrated with case studies. A comparison of various MPC schemes is presented, showcasing that the implementation using criterion $\mathcal{K}$ results in intensified processes kept stable at all times, whilst reducing computational cost with regards to standard nonlinear MPC schemes. Furthermore, reaction times are reduced by at least two-fold with respect to processes run at constant temperatures. Keywords: Thermal stability criterion, Model predictive control, Process intensification, Batch reactors

\footnotetext{
${ }^{*}$ Corresponding Author

Email address: vsv20@cam.ac.uk (Vassilios S. Vassiliadis)
} 
${ }_{27}$ Roman Symbols

28 Symbol

${ }_{29} \quad A$

$30 \quad[\mathrm{~A}],[\mathrm{B}],[\mathrm{C}]$

$31 \quad B$

${ }_{32} C_{p}, C_{p \mathrm{C}}$

${ }_{33} D a$

${ }_{34} \Delta H_{r}$

${ }_{35} E_{a}$

36 $f$

$37 \quad h$

$38 \quad \mathbf{J}$

$39 \quad J_{z l}$

${ }_{40} \quad k_{0}$

${ }_{41} m_{B}, m_{D a_{\mathrm{res}}}, m_{\gamma}, m_{S t}$

${ }_{42} \quad N$

${ }_{43} n_{\mathrm{A}}, n_{\mathrm{B}}$

${ }_{44} \quad q_{\mathrm{C}}$

${ }_{45} Q_{\text {gen }}$

${ }_{46} \quad R$

${ }_{47} r$

${ }_{48} \quad R e$

$49 \quad S t$

\section{Description}

heat transfer coefficient area $\left[\mathrm{m}^{2}\right]$

concentration of component $\mathrm{A}, \mathrm{B}$ and $\mathrm{C}$, respectively $\left[\mathrm{kmol} \mathrm{m}^{-3}\right]$

Barkelew number $[-]$

heat capacity of reaction mixture and coolant, respectively $\left[\mathrm{kJkg}^{-1} \mathrm{~K}^{-1}\right]$

Damköhler number $[-]$

enthalpy of reaction $\left[\mathrm{kJmol}^{-1}\right]$

activation energy of the reaction $\left[\mathrm{Jmol}^{-1}\right]$

nonlinear function for differential equation [-]

equations for physical properties [-]

Jacobian matrix [-]

Jacobian matrix entry in row $z$, column $l\left[\mathrm{~s}^{-1}\right]$

pre-exponential Arrhenius constant for the reaction $\left[\left(\mathrm{m}^{3} \mathrm{kmol}^{-1}\right)^{n-1} \mathrm{~s}^{-1}\right]$

stability criterion coefficients [-]

number of differential equations $[-]$

reaction orders of components $\mathrm{A}$ and $\mathrm{B}$, respectively [-]

volumetric folw rate of coolant $\left[\mathrm{m}^{3} \mathrm{~s}^{-1}\right]$

heat generation by exothermic reaction $\left[\mathrm{J} \mathrm{s}^{-1}\right]$

universal molar gas constant $\left[\mathrm{Jmol}^{-1} \mathrm{~K}^{-1}\right]$

reaction rate $\left[\mathrm{kmol} \mathrm{m}^{-3} \mathrm{~s}^{-1}\right]$

Reynolds number in reactor $[-]$

Stanton number $[-]$ 
${ }_{51} t_{c}, t_{p}$

${ }_{52} T_{\mathrm{R}}, T_{\mathrm{C}}, T_{\mathrm{sp}}$

53

${ }_{54} \quad t_{\text {ref }}$

${ }_{55} U$

${ }_{56} V_{\mathrm{R}}, V_{\mathrm{C}}$

$57 \quad x$

${ }_{58} y_{j}, \bar{y}_{j}, \hat{y}_{j}$

59

60 Greek Symbols

61 Symbol

${ }_{62} \epsilon_{t o l}$

${ }_{63} \gamma$

${ }_{64} \lambda_{j}$

${ }_{65} \mu_{j}$

${ }_{66} \nu_{\mathrm{A}}, \nu_{\mathrm{B}}$

$67 \quad \Phi$

${ }_{68} \rho, \rho_{\mathrm{C}}$

${ }_{69} \varepsilon_{\text {div }}$

70 Superscripts

71 Symbol

${ }_{72} i$

73 Other Symbols time of simulation $[\mathrm{s}]$

control and prediction horizon for MPC (s)

temperature of reactor contents, coolant and set point reaction set point, respectively $[\mathrm{K}]$

reference time for divergence of Jacobian $[\mathrm{s}]$

heat transfer coefficient $\left[W \mathrm{~m}^{-2}, \mathrm{~K}^{-1}\right]$

volume of the reactor and the cooling jacket, respectively $\left[\mathrm{m}^{3}\right]$

differential variable $[-]$

mass fraction, mole fraction and volume fraction of component $j$, respectively $[-]$

\section{Description}

ODE solver tolerance [-]

Arrhenius number $[-]$

thermal conductivity of component $j\left[\mathrm{~W} \mathrm{~m}^{-1} \mathrm{~K}^{-1}\right]$

viscosity of component $j[\mathrm{Pas}]$

stoichiometric coefficients of components A and B [-]

objective function for MPC algorithm [-]

density of reactor contents and coolant, respectively $\left[\mathrm{kgm}^{-3}\right]$

error of the divergence $\left[\mathrm{s}^{-1}\right]$

\section{Description}

time step of simulation $[-]$ 


\section{Symbol Description}

divergence estimate at boundary of stability $\left[\mathrm{s}^{-1}\right]$

stability criterion $\left[\mathrm{s}^{-1}\right]$

\section{Introduction}

The loss of thermal stability in chemical reactions leads to an uncontrolled increase in reaction temperature which can cause significant safety issues, an increased downtime of reactors and hence high financial loss. In batch processes thermal runaways can occur which then require the reactions to be stopped by inhibitors, making the product unsellable. If no such action is taken an explosion or uncontrolled discharge of chemicals can result, bearing high risks for the health of workers and the environment.

Model Predictive Control (MPC) is an advanced control scheme within which the control variables of the system are optimised whilst considering system constraints. It is common in literature to use a linearisation of the system present. This enables the application of linear MPC schemes (Rawlings and Mayne, 2015; Ellis et al., 2014; Haber et al., 2011; Mayne and Michalska, 1990). For linear MPC schemes the closed-loop stability can be proven theoretically by using Lyapunov functions (Huang et al., 2012; DeHaan and Guay, 2010). End-point constraints are often employed for a very large prediction horizon if such a Lyapunov function cannot be found. Larger time frames are necessary for complex and highly nonlinear systems which leads to higher computational cost in order to guarantee stability. If the stability of the system can be quantified by a criterion, this can reduce the time frame of simulation used and hence reduce computational time.

Previous work on MPC for batch reactors considered applying linearised MPC schemes in which the linear models are constantly updated during the process with continuous parameter estimation (Nagy and Braatz, 2003; Kalmuk et al., 2017), or offline step response model identification to model the system correctly at each operating point (Kufoalor et al., 2015). Shrinking horizon MPC schemes were introduced in literature which optimise the batch process over the whole process duration, hence proving to give stable operation but resulting in very large optimisation problems with large computational cost (Simon et al., 2008). Furthermore, MPC schemes were introduced in literature which make use of neural network models of the system dynamics, which are then used for the optimisation stage within MPC (Hosen et al., 2011). These approaches do not consider the thermal runaway behaviour of batch processes specifically but assume that it can be dealt with by approximation of the system dynamics around a nominal operating point. In this work an alternative approach is 
presented which makes use of the full nonlinear dynamic model, obtained from first principles, to find the best inputs to the batch reactor system.

The application of MPC with an integrated stability criterion enables a safer process control and the advantageous possibility of increasing the efficiency of exothermic chemical batch processes. For the application of MPC to chemical reactors, accurate process models are required. Hence stability criteria with as little computational cost as possible are of profound importance, as detailed process models require high computational time.

The theory on thermal explosions (Semenov, 1940) characterises the change in stability of stationary processes with simple reaction kinetics and is not adequate for the analysis of dynamic systems.

The Routh-Hurwitz Criterion (Anagnost and Desoer, 1991; Stephanopoulos, 1984; Hurwitz, 1895; Routh, 1877) is commonly used to quantify the stability of operating points for continuous steady-state systems. This criterion requires that the dynamics can be linearised close to such operating points. This cannot be done for strongly nonlinear batch processes, as wrong predictions of system dynamics are obtained with such models. Hence it is not applicable to the systems considered in this paper.

The Lyapunov exponent method enables to quantify the chaotic nature of processes (Melcher, 2003; Strozzi and Zaldívar, 1994). The convergent or divergent nature of highly nonlinear processes can be reliably predicted with this method. This characterisation requires to simulate the nonlinear system for each differential variable for a given time frame, which ideally should be infinitely large. Therefore, the evaluation of Lyapunov exponents for nonlinear systems with many variables can be very expensive, which limits the applicability to MPC schemes which require low computational cost. Nevertheless, its reliability at predicting system stability is a key advantage for a potential MPC implementation.

The divergence method (Strozzi and Zaldívar, 1999; Arnold, 1973) also derives from chaos theory; compared to Lyapunov exponents it does not describe the transition to instability in a reliable manner. In Kähm and Vassiliadis (2018) it was shown that using the divergence criterion to detect thermal runaways in the exothermic batch reactions is not feasible. In this work it is shown that for more complex reaction kinetics the divergence criterion is not feasible for the detection of thermal stability either. Hence, the implementation of the divergence criterion to MPC schemes leads to much less efficient processes.

In Rossi et al. (2015) a stability criterion was used to give a different advanced control scheme. A Boolean variable which gives rise to the system stability is determined by an algorithm. Similar to logarithmic barrier functions, this Boolean variable comes into effect within the objective function if the process enters an unstable operating regime. The function defining this Boolean variable is system-specific, which leads to large implementation costs 
for new systems. As for other penalty function methods, this approach can also lead to badly scaled problems. It was tested if including the stability criterion within the objective function would result in better control. The resulting problem, as expected, turned out to be badly scaled and hence was deemed as not feasible.

The criterion for thermal stability introduced in Kähm and Vassiliadis (2018) enables the efficient control of exothermic batch processes with small computational cost for the implementation with MPC. The criterion of thermal stability $\mathcal{K}$ was developed for exothermic batch reactions with overall reaction orders of 1 to 3 , in which the reaction rate depends solely on the concentration of one component.

The aims of this paper are twofold. The first aim is the extension of stability criterion $\mathcal{K}$ for exothermic batch processes with a single reaction composed of two reactants.

The kinetics of the analysed chemical reaction scheme have the following properties:

- the kinetics depend on the concentration of both reaction components

- the reactants each have varying stoichiometric coefficients

- the reaction order for each reaction component varies between 1 and 4

The second aim of this paper is to improve the efficiency of batch processes with the use of stability criterion $\mathcal{K}$ implemented with MPC for all the above reaction schemes. Of major importance for the implementation is computational cost and reliability.

The paper is organised as follows: in Section 2 the process models for each reaction scheme considered, together with mass and energy balances are presented. In Section 3 stability criteria found in literature are presented and assessed in terms of their feasibility of being implemented with MPC. In Section 4 stability criterion $\mathcal{K}$, based on the divergence criterion, is derived and the logic behind it is explained. The coefficients giving rise to criterion $\mathcal{K}$ are presented and the resulting stability criterion profiles are shown for each process. In Section 5 process intensification based on criterion $\mathcal{K}$ embedded in a standard MPC scheme for exothermic batch processes is presented. This novel control scheme is compared to standard control schemes in terms of stability and computational cost. A detailed comparison of each implementation is carried out to give recommendations for potential use in industry. In Section 6 the results of this paper are summarised and possibilities for future work are discussed.

\section{Process model}

\subsection{Mass and energy balances for batch reactors}

The batch reactor system considered in the following simulations is shown in Figure 1. 


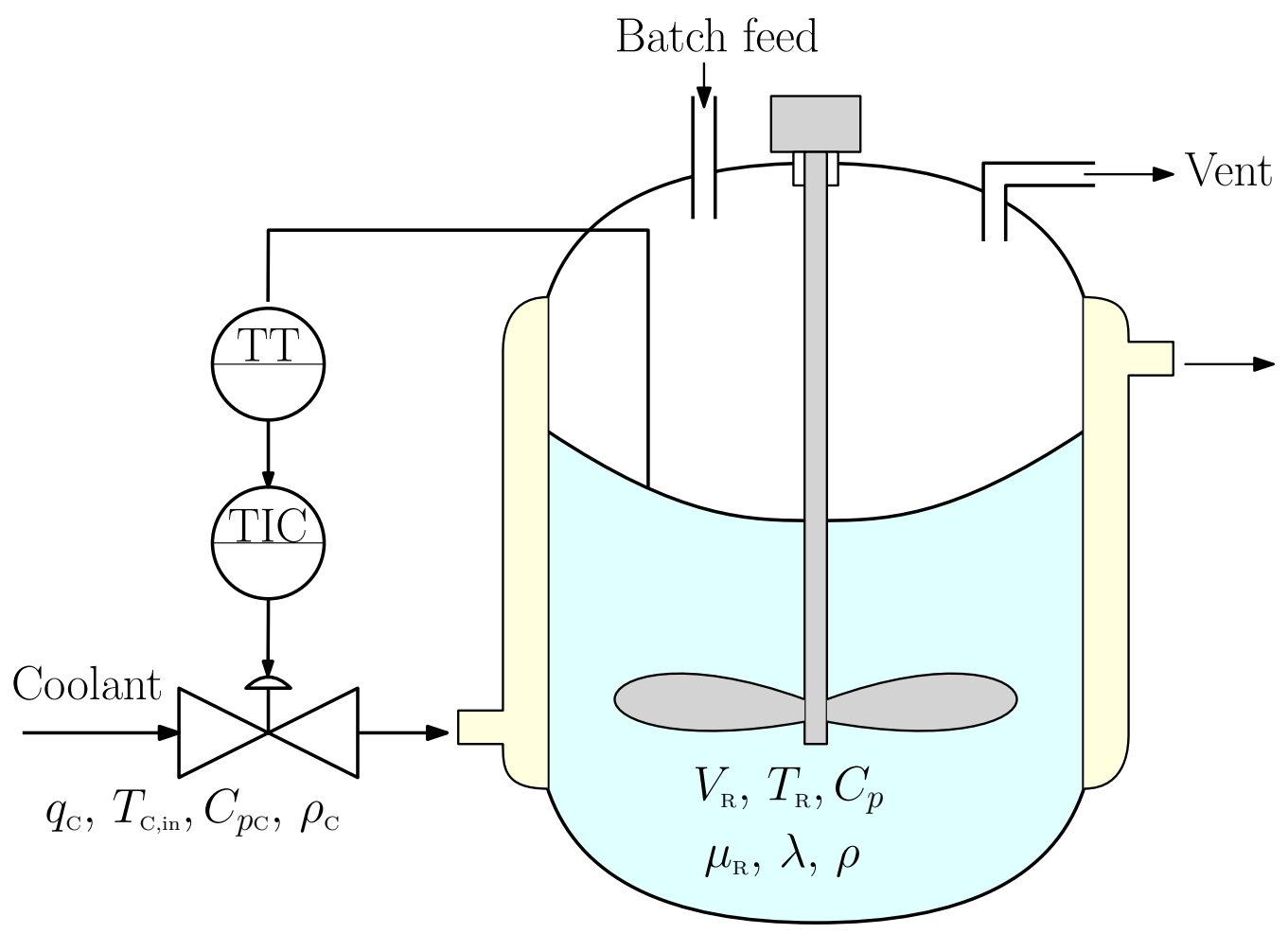

Figure 1: Batch reactor diagram for simulated systems.

The overall mass balance of the reactor contents with respect to time $t$ is given by:

$$
\frac{\mathrm{d}\left(\rho V_{\mathrm{R}}\right)}{\mathrm{d} t}=0
$$

where $V_{\mathrm{R}}$ is the reactor volume and $\rho$ is the reacting mixture density.

The following reaction is considered to occur within the batch reactor:

$$
\nu_{\mathrm{A}} \mathrm{A}+\nu_{\mathrm{B}} \mathrm{B} \rightarrow \mathrm{C}
$$

where $\nu_{\mathrm{A}}$ and $\nu_{\mathrm{B}}$ are the stoichiometric coefficients of reactants $\mathrm{A}$ and $\mathrm{B}$.

Therefore the mass of the three components $\mathrm{A}, \mathrm{B}$ and $\mathrm{C}$ have to be known. The mass balance for each reagent and product is given by:

$$
\begin{aligned}
& \frac{\mathrm{d}[\mathrm{A}]}{\mathrm{d} t}=-r \\
& \frac{\mathrm{d}[\mathrm{B}]}{\mathrm{d} t}=-r \\
& \frac{\mathrm{d}[\mathrm{C}]}{\mathrm{d} t}=r
\end{aligned}
$$

where $r$ is the reaction rate, presented in the following section. 
The energy balance of the reaction mixture is given by:

$$
\frac{\mathrm{d}}{\mathrm{d} t}\left(\rho V_{\mathrm{R}} C_{p} T_{\mathrm{R}}\right)=r\left(-\Delta H_{r}\right) V_{\mathrm{R}}-U A\left(T_{\mathrm{R}}-T_{\mathrm{C}}\right)
$$

where $C_{p}$ is the reaction mixture heat capacity, $\Delta H_{r}$ is the reaction enthalpy, $U$ is the heat transfer coefficient from reactor contents to the cooling jacket, $A$ is the heat transfer area of the cooling jacket, and $T_{C}$ is the coolant temperature.

The energy balance for the cooling jacket is given by:

$$
\frac{\mathrm{d}}{\mathrm{d} t}\left(V_{\mathrm{C}} \rho_{\mathrm{C}} C_{p \mathrm{C}} T_{\mathrm{C}}\right)=q_{\mathrm{C}} \rho_{\mathrm{C}} C_{p \mathrm{C}}\left(T_{\mathrm{C}, \text { in }}-T_{\mathrm{C}}\right)+U A\left(T_{\mathrm{R}}-T_{\mathrm{C}}\right)
$$

where $V_{\mathrm{C}}$ is the cooling jacket volume, $\rho_{\mathrm{C}}$ is the coolant density, $C_{p \mathrm{C}}$ is the coolant heat capacity and $T_{\mathrm{C}, \text { in }}$ is the coolant inlet temperature.

\subsection{Reaction kinetics}

The reactions analysed in this work occur in a homogeneous liquid phase. Furthermore, the reactions are assumed to be irreversible and exothermic. The reaction scheme is given by a single reaction, given in Equation 2.2.

The rate of reaction can be described with an Arrhenius expression (Davis and Davis, 2003), including the order of reaction $n_{\mathrm{A}}$ and $n_{\mathrm{B}}$ with respect to reactants $\mathrm{A}$ and $\mathrm{B}$, respectively. This expression is given by:

$$
r=k_{0} \exp \left(-\frac{E_{a}}{R T_{\mathrm{R}}}\right) \times[\mathrm{A}]^{n_{\mathrm{A}}}[\mathrm{B}]^{n_{\mathrm{B}}}
$$

where $[\mathrm{A}]$ and $[\mathrm{B}]$ are the concentrations of components $\mathrm{A}$ and $\mathrm{B}, E_{a}$ is the activation energy of the reaction, $T_{\mathrm{R}}$ is the reactor temperature, $R$ is the universal molar gas constant and $k_{0}$ is the pre-exponential Arrhenius constant.

\subsection{Process parameters}

The parameters specific to the reaction kinetics and energy produced are varied to get a range of process scenarios, for which the stability is analysed. The different processes are denoted e.g. by $\mathrm{P}_{12}$ or $\mathrm{P}_{5}$, corresponding to process 12 and 5 for the reaction scheme above, respectively. Below the various process parameters are shown.

The reaction scheme considered in this work corresponds to a more complex kinetic scheme than that presented in Kähm and Vassiliadis (2018). Therefore, 20 different process are considered for this reaction scheme. The process parameters are summarised in Table 1. 
Table 1: Process parameters for the reaction scheme.

\begin{tabular}{|c|c|c|c|c|c|c|c|c|}
\hline Process & $\begin{array}{c}k_{0} \times 10^{-3} \\
{\left[\frac{\mathrm{m}^{3(n-1)}}{\mathrm{kmol}^{(n-1)} \mathrm{s}}\right]^{*}}\end{array}$ & $\begin{array}{l}\Delta H_{r} \\
{\left[\frac{\mathrm{kJ}}{\mathrm{mol}}\right]}\end{array}$ & $\begin{array}{l}n_{\mathrm{A}} \\
{[-]}\end{array}$ & $\begin{array}{l}n_{\mathrm{B}} \\
{[-]}\end{array}$ & $\begin{array}{l}{[\mathrm{A}]_{0}} \\
{\left[\frac{\mathrm{kmol}}{\mathrm{m}^{3}}\right]}\end{array}$ & $\begin{array}{l}\nu_{\mathrm{A}} \\
{[-]}\end{array}$ & $\begin{array}{l}\nu_{\mathrm{B}} \\
{[-]}\end{array}$ & $\begin{array}{c}E_{a} / R \\
{[\mathrm{~K}]}\end{array}$ \\
\hline $\mathrm{P}_{1}$ & 10 & -150 & 1.0 & 1.0 & 10.0 & 1.0 & 1.0 & 9525 \\
\hline $\mathrm{P}_{2}$ & 3.0 & -110 & 2.0 & 2.0 & 10.0 & 1.0 & 1.0 & 9525 \\
\hline $\mathrm{P}_{3}$ & 60 & -110 & 1.5 & 1.0 & 10.0 & 1.0 & 1.0 & 9525 \\
\hline $\mathrm{P}_{4}$ & 80 & -110 & 1.0 & 1.5 & 10.0 & 1.0 & 1.5 & 9525 \\
\hline $\mathrm{P}_{5}$ & 120 & -150 & 1.0 & 1.0 & 8.0 & 1.0 & 1.0 & 9400 \\
\hline $\mathrm{P}_{6}$ & 50 & -150 & 1.5 & 1.0 & 8.0 & 1.0 & 1.0 & 9400 \\
\hline $\mathrm{P}_{7}$ & 23 & -130 & 1.5 & 1.5 & 8.0 & 1.0 & 1.0 & 9450 \\
\hline $\mathrm{P}_{8}$ & 20 & -140 & 2.0 & 1.0 & 8.0 & 1.0 & 1.0 & 9450 \\
\hline $\mathrm{P}_{9}$ & 5.0 & -110 & 2.0 & 2.0 & 8.0 & 1.0 & 1.0 & 9525 \\
\hline$P_{10}$ & 90 & -130 & 1.5 & 1.0 & 8.0 & 2.0 & 1.0 & 9525 \\
\hline$P_{11}$ & 100 & -130 & 1.5 & 1.0 & 8.0 & 2.0 & 1.5 & 9525 \\
\hline $\mathrm{P}_{12}$ & 125 & -150 & 1.5 & 1.0 & 6.0 & 1.5 & 1.5 & 9525 \\
\hline $\mathrm{P}_{13}$ & 30 & -150 & 2.5 & 1.0 & 6.0 & 1.0 & 2.0 & 9700 \\
\hline $\mathrm{P}_{14}$ & 5.0 & -180 & 3.5 & 1.0 & 6.0 & 1.5 & 2.5 & 9650 \\
\hline $\mathrm{P}_{15}$ & 1.5 & -280 & 4.0 & 1.0 & 6.0 & 2.5 & 2.5 & 9670 \\
\hline $\mathrm{P}_{16}$ & 110 & -150 & 1.0 & 1.5 & 5.0 & 1.0 & 1.0 & 9525 \\
\hline $\mathrm{P}_{17}$ & 80 & -150 & 1.0 & 1.5 & 5.0 & 1.0 & 1.0 & 9350 \\
\hline $\mathrm{P}_{18}$ & 120 & -150 & 1.0 & 1.5 & 5.0 & 1.0 & 1.0 & 9550 \\
\hline $\mathrm{P}_{19}$ & 120 & -140 & 1.0 & 1.5 & 5.0 & 1.0 & 1.0 & 9480 \\
\hline $\mathrm{P}_{20}$ & 120 & -140 & 1.0 & 1.5 & 5.0 & 1.0 & 1.0 & 9500 \\
\hline
\end{tabular}

The initial concentration of component $\mathrm{B}$, and the initial temperature of the reactor are held constant for all the above processes. These are set to $[\mathrm{B}]_{0}=8.0 \mathrm{kmolm}^{-3}$ and $T_{\mathrm{R} 0}=405 \mathrm{~K}$.

The stirrer used in this model is assumed to result in a turbulent mixing of the reactor contents with a Reynolds number of $R e=10^{5}$. Hence, the concentration and temperature of the reacting mixture only varies across an insignificant boundary layer at the reactor walls. Therefore, using uniform reactor properties (ideal mixing) is a fair assumption.

The changes in viscosity and specific heat capacity of the reaction mixture are evaluated according to the composition, together with physical data given in Table 2. 
Table 2: Physical properties of components A, B and C.

\begin{tabular}{ccccc}
\hline Physical property & $\begin{array}{c}\rho \\
{\left[\mathrm{kg} \mathrm{m}^{-3}\right]}\end{array}$ & $\begin{array}{c}\mu \\
{\left[\mathrm{Pas}^{-1}\right]}\end{array}$ & $\begin{array}{c}C_{p} \\
{\left[\mathrm{Jkg}^{-1} \mathrm{~K}^{-1}\right]}\end{array}$ & $\begin{array}{c}\lambda \\
{\left[\mathrm{Wm}^{-1} \mathrm{~K}^{-1}\right]}\end{array}$ \\
\hline Component & & & & \\
A & 911 & $1.00 \times 10^{-4}$ & 1100 & 0.300 \\
B & 790 & $3.00 \times 10^{-4}$ & 950 & 0.250 \\
$\mathrm{C}$ & 1200 & $9.00 \times 10^{-4}$ & 850 & 0.150 \\
\hline
\end{tabular}

The changes in density, viscosity and heat capacity of the reaction mixture with changing temperature and composition are approximated in the simulation. Depending on the composition the following equations are used to estimate the physical properties of the reaction mixture:

$$
\begin{aligned}
\frac{1}{\rho} & =\sum_{j} y_{j} / \rho_{j} \\
\ln \mu & =\sum_{j} \bar{y}_{j} \ln \mu_{j} \\
C_{p} & =\sum_{j} y_{j} C_{p j} \\
\lambda & =\sum_{j} \hat{y}_{j} \lambda_{j}
\end{aligned}
$$

where $y_{j}$ is the mass fraction, $\bar{y}_{j}$ is the molar fraction, and $\hat{y}_{j}$ is the volume fraction of component $j$. These equations are obtained from Hirschfelder et al. (1955), Teja (1983) and Green and Perry (2008).

The accurate description of the temperature and composition relationships for liquid mixtures is very difficult. Hence, for the change in temperature linear interpolation of tabulated physical properties for water, ethylene oxide and ethylene glycol, components A, B and C respectively, are used. The temperature dependence of the above parameters is obtained from Dever et al. (2004), Crittenden et al. (2012) and Bohne et al. (2010).

The heat transfer coefficient $U$ of the reaction mixture to the cooling jacket is evaluated from the properties of the reaction mixture and the coolant, as well as the flow rate of coolant (Sinnot, 2005).

\subsection{Reactor parameters}

The chemical reactor models simulated have a cooling/heating jacket, as can be seen in Figure 1, which controls the reactor temperature by varying the coolant flow rate. A stirrer in each reactor is assumed to be ideal in that all reactor properties are uniform within the reaction mixture. The coolant flow rate is controlled by either a PI controller or by MPC. 
The reactor properties for each size of reactor are shown in Table 3.

Table 3: Reactor properties used for simulations.

\begin{tabular}{cccccc}
\hline Parameter & $\begin{array}{c}V_{\mathrm{R}} \\
{\left[\mathrm{m}^{3}\right]}\end{array}$ & $\begin{array}{c}V_{\mathrm{C}} \\
{\left[\mathrm{m}^{3}\right]}\end{array}$ & $\begin{array}{c}A \\
{\left[\mathrm{~m}^{2}\right]}\end{array}$ & $\begin{array}{c}q_{\mathrm{C}, \max } \\
{\left[\mathrm{m}^{3} \mathrm{~s}^{-1}\right]}\end{array}$ & $\begin{array}{c}T_{\mathrm{C}, \text { in }} \\
{[\mathrm{K}]}\end{array}$ \\
\hline $\mathrm{P}_{1}-\mathrm{P}_{5}$ & 32 & 2.0 & 49 & 0.060 & 300 \\
$\mathrm{P}_{6}-\mathrm{P}_{10}$ & 20 & 1.4 & 36 & 0.043 & 300 \\
$\mathrm{P}_{11}-\mathrm{P}_{15}$ & 8 & 0.5 & 20 & 0.023 & 300 \\
$\mathrm{P}_{16}-\mathrm{P}_{20}$ & 0.8 & 0.17 & 4.2 & 0.005 & 300 \\
\hline
\end{tabular}

The verification of stability criterion $\mathcal{K}$ requires a transition from stable to unstable operation. Hence a PI controller with fast control is used, the parameters of which are obtained by trial and error. The standard form of PI controllers is given in Stephanopoulos (1984). The parameters of the PI controller used are given in Table 4.

Table 4: Parameters for PI controller used in case studies.

\begin{tabular}{cc}
\hline Parameter & Value \\
\hline Proportional (P), $K_{p}$ & $10 \mathrm{~m}^{3} \mathrm{~s}^{-1} \mathrm{~K}^{-1}$ \\
Integral (I), $\tau_{I}$ & $1000 \mathrm{Ks}^{2} \mathrm{~m}^{-3}$ \\
\hline
\end{tabular}

All simulations presented for this work were done with an HP EliteDesk 800 G2 Desktop Mini PC with an Intel ${ }^{\circledR}$ Core $^{\mathrm{TM}} 3.20 \mathrm{GHz}$ i5-65000 processor with 16 GB RAM. The operating system was Windows 7 Enterprise. The computational language used is MATLAB ${ }^{\mathrm{TM}}$, with the readily available algorithm ode15s(Shampine et al., 1999) for dynamic simulations. Due to its simplicity of developing code, MATLAB ${ }^{\mathrm{TM}}$ was used instead of more efficient programming languages as $\mathrm{C}, \mathrm{C}++$ and FORTRAN.

\section{Analysis of stability criteria}

In the Introduction the Lyapunov exponent method and the divergence method were identified as the most promising techniques to analyse the thermal stability of exothermic batch processes when embedded with MPC. The advantages and disadvantages of both methods are examined in this section.

\subsection{Lyapunov exponent method}

The theory on Lyapunov exponents was derived from chaos theory (Strozzi and Zaldívar, 1994; Melcher, 2003; van der Kloet and Neerhoff, 2003). For chaotic systems it can be determined if the trajectory of system variables diverge or converge when experiencing a 
small initial perturbation. Hence, each system variable gives rise to a Lyapunov exponent, denoted by $\Lambda$.

As was discussed in Kähm and Vassiliadis (2018), the time frame required to evaluate Lyapunov exponents, as well as the initial perturbation $\varepsilon$, need careful tuning. In order to not overlook a thermal runaway it is necessary to check the stability for many values of final time $t_{f}$, leading to to a large number of simulations. Hence this results in high computational time. Further work is necessary to reduce the computational time of this method to make it viable for online MPC schemes.

\subsection{Divergence method}

A general set of nonlinear differential equations is given by:

$$
\begin{aligned}
\dot{x}_{1} & =f_{1}(x, t) \\
\vdots & \vdots \\
\dot{x}_{N} & =f_{N}(x, t)
\end{aligned}
$$

where $N$ is the number of differential variables $\dot{x}$, and $f(x, t)$ is a general nonlinear function. Using a Taylor series for a first order approximation yields:

$$
\dot{x}=\mathbf{J} x
$$

where $\mathbf{J}$ is the Jacobian matrix including all first order derivatives. The entry at row $z$ and column $l, J_{z l}$, is evaluated by:

$$
J_{z l}=\frac{\partial f_{z}}{\partial x_{l}}
$$

To detect a thermal runaway, only the diagonal entries of the Jacobian with respect to the variables contributing towards the heat of reaction are required (Copelli et al., 2014; Bosch et al., 2004; Kähm and Vassiliadis, 2018).

The heat generation $Q_{g e n}$ in the reactor is given by:

$$
Q_{\text {gen }}=\sum_{z} r_{z}\left(-\Delta H_{r_{z}}\right) V_{\mathrm{R}}
$$

Hence the state variables of interest are the concentrations in each reaction rate $r_{z}$, as well as the reactor temperature $T_{\mathrm{R}}$.

A more detailed derivation of the divergence of the Jacobian matrix and the divergence criterion is shown in Kähm and Vassiliadis (2018). 
3.2.1. Jacobian matrix derivation for batch reaction model

The relevant Jacobian matrix entries of the relevant variables and system Equations (2.1) (2.7) lead to the following expression:

$$
\begin{aligned}
\operatorname{div}[\mathbf{J}] \times t_{\text {ref }}= & -\nu_{\mathrm{A}} n_{\mathrm{A}} k_{0} \exp \left(-\frac{E_{a}}{R T_{\mathrm{R}}}\right) \times[\mathrm{A}]^{n_{\mathrm{A}}-1}[\mathrm{~B}]^{n_{\mathrm{B}}} \\
& -\nu_{\mathrm{B}} n_{\mathrm{B}} k_{0} \exp \left(-\frac{E_{a}}{R T_{\mathrm{R}}}\right) \times[\mathrm{A}]^{n_{\mathrm{A}}}[\mathrm{B}]^{n_{\mathrm{B}}-1} \\
& +\frac{1}{\rho C_{p} V_{\mathrm{R}}}\left[\frac{E_{a}}{R T_{\mathrm{R}}^{2}} k_{0} \exp \left(-\frac{E_{a}}{R T_{\mathrm{R}}}\right)\right. \\
& \left.\times[\mathrm{A}]^{n_{\mathrm{A}}}[\mathrm{B}]^{n_{\mathrm{B}}}\left(-\Delta H_{r}\right) V_{\mathrm{R}}-U A\right] \\
\operatorname{div}[\mathbf{J}] \times t_{\mathrm{ref}}= & -\left(\nu_{\mathrm{A}} n_{\mathrm{A}} D a_{\mathrm{A}}+\nu_{\mathrm{B}} n_{\mathrm{B}} D a_{\mathrm{B}}\right) \times \exp (-\gamma) \\
& +B \gamma D a_{\mathrm{A}} \exp (-\gamma)-S t
\end{aligned}
$$

where

$$
\begin{aligned}
B & =\frac{[\mathrm{A}]\left(-\Delta H_{r}\right)}{\rho C_{p} T_{\mathrm{R}}} \\
\gamma & =\frac{E_{a}}{R T_{\mathrm{R}}} \\
D a_{\mathrm{A}} & =k_{0}[\mathrm{~A}]^{n_{\mathrm{A}}-1}[\mathrm{~B}]^{n_{\mathrm{B}}} t_{\mathrm{ref}} \\
D a_{\mathrm{B}} & =k_{0}[\mathrm{~A}]^{n_{\mathrm{A}}}[\mathrm{B}]^{n_{\mathrm{B}}-1} t_{\mathrm{ref}} \\
S t & =\frac{U A}{\rho C_{p} V_{\mathrm{R}}} t_{\mathrm{ref}}
\end{aligned}
$$

where $\mathrm{B}$ is the Barkelew number, $\gamma$ is the Arrhenius number, $D a_{\mathrm{A}}$ and $D a_{\mathrm{B}}$ are the Damkóhler numbers for components A and B, and $S t$ is the Stanton number.

From Equation (3.6) it can be seen that the divergence only depends on the stoichiometric coefficients, the reaction orders, and the dimensionless numbers given in Equations $(3.7 a)-$ (3.7e). The following analysis shows that the value of $t_{\text {ref }}$ has no influence on the value of the divergence of the Jacobian matrix as it cancels out.

\subsubsection{Case studies with divergence criterion}

The temperature profiles together with the respective divergence profiles for processes $\mathrm{P}_{1}-\mathrm{P}_{10}$ are shown in Figures 3 and 5. The temperature set point is increased in two stages: initially a stable process is present. As the temperature of the system increases, the processes becomes uncontrollable, as the cooling water capacity is not sufficient to keep the process under control. After the second increase in temperature a thermal runaway occurs. Similar 
temperature and divergence profiles are obtained for processes $\mathrm{P}_{11}-\mathrm{P}_{20}$. Hence, for clarity, these graphs are not explicitly shown here. The temperature and divergence profiles for processes $\mathrm{P}_{1}-\mathrm{P}_{5}$ are shown in Figure 2 and Figure 3.

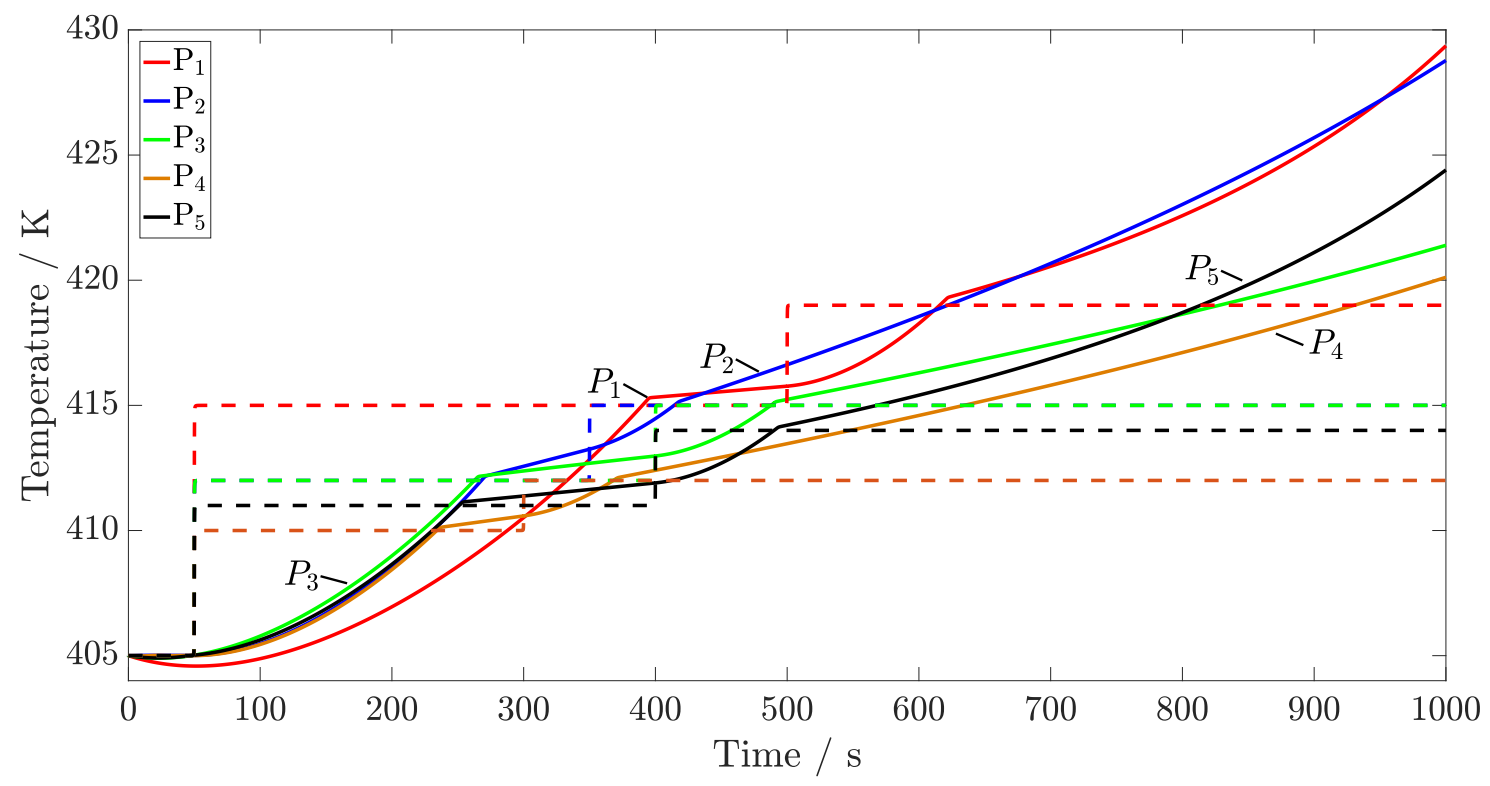

Figure 2: Temperature profiles for processes $\mathrm{P}_{1}-\mathrm{P}_{5}$.

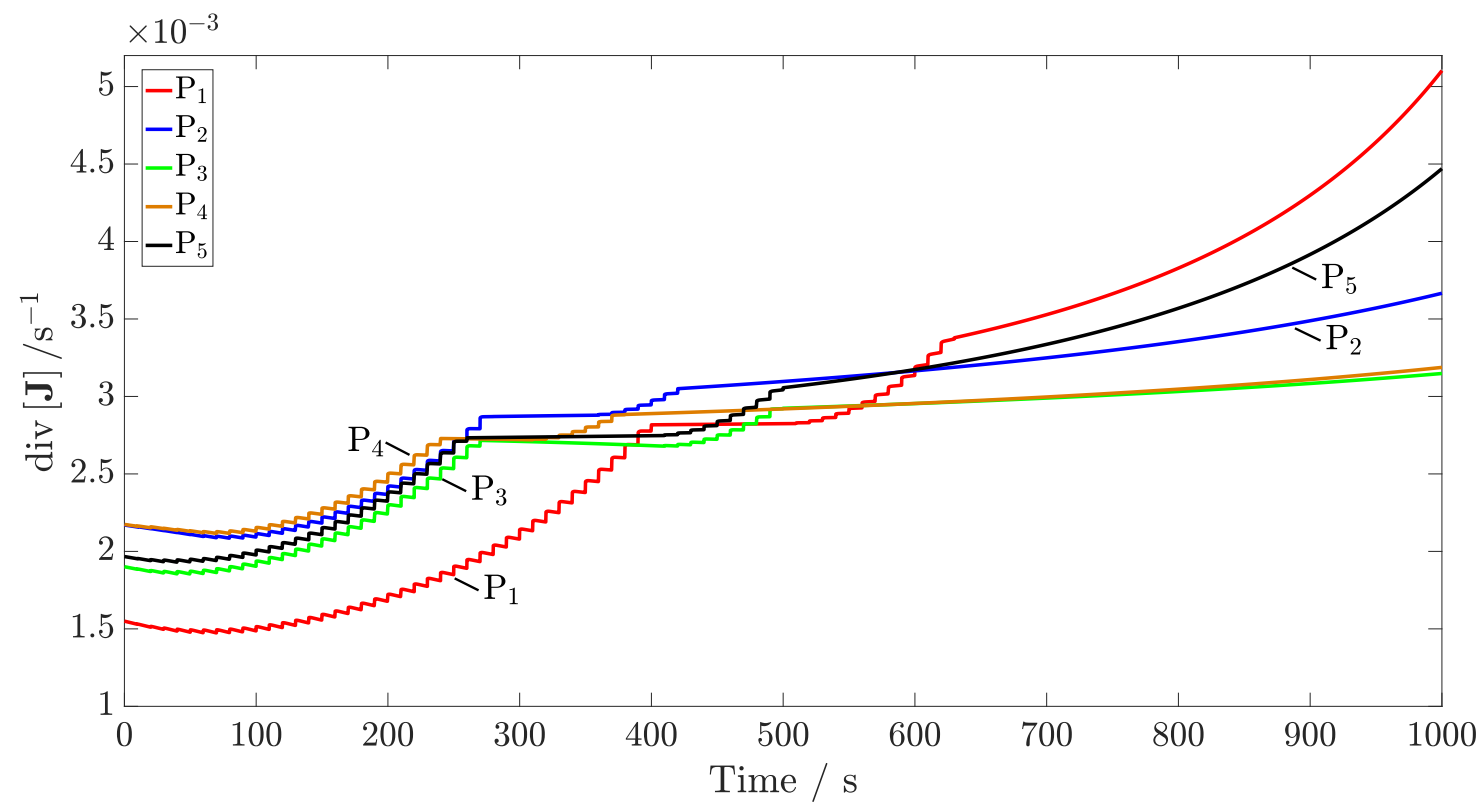

Figure 3: Divergence profiles for processes $\mathrm{P}_{1}-\mathrm{P}_{5}$.

For processes $\mathrm{P}_{1}-\mathrm{P}_{5}$ the divergence profiles follow a similar trajectory as the respective temperature profiles. After loss of stability the divergence of the Jacobian matrix, $\operatorname{div}[\mathbf{J}]$, 

gence profiles for processes $\mathrm{P}_{6}-\mathrm{P}_{10}$ are shown in Figures 4 and 5 .

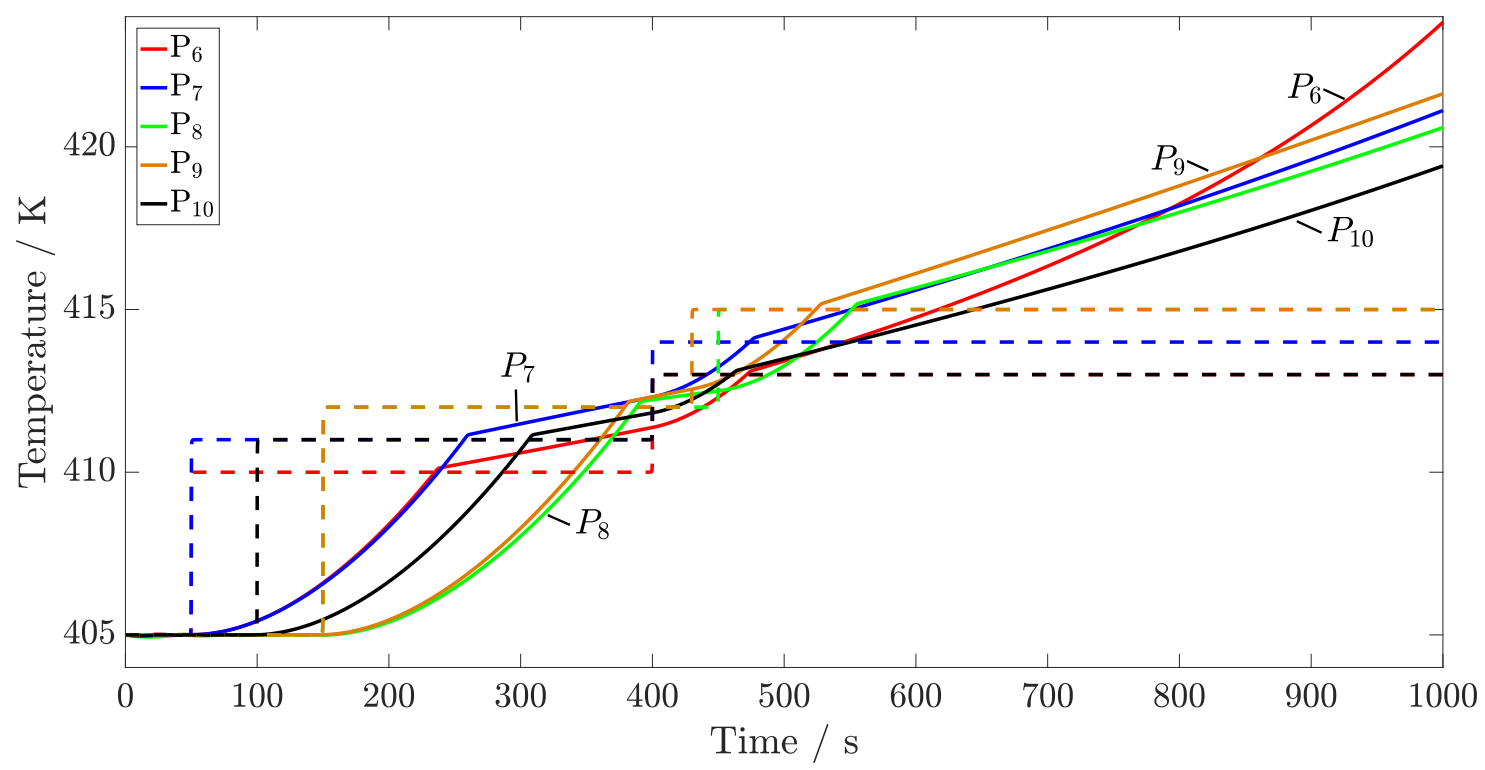

Figure 4: Temperature profiles for processes $\mathrm{P}_{6}-\mathrm{P}_{10}$.

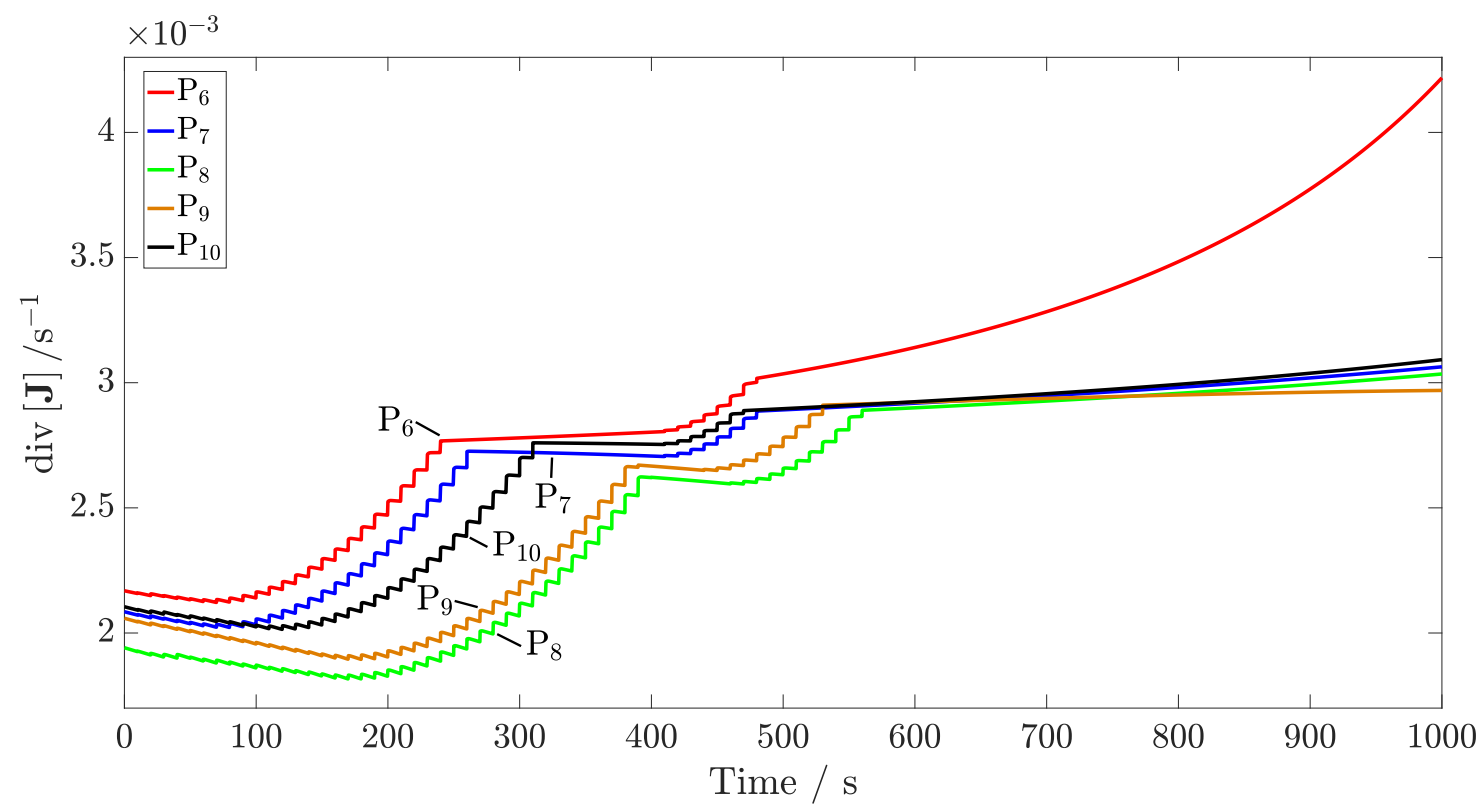

Figure 5: Divergence profiles for processes $\mathrm{P}_{6}-\mathrm{P}_{10}$.

increases once the temperature of the system starts increasing. The temperature and diver3 p

In processes $\mathrm{P}_{6}-\mathrm{P}_{10}$ the divergence $\operatorname{div}[\mathbf{J}]$ increases significantly once a thermal runaway can be observed from the respective temperature profiles. The temperature and divergence profiles for processes $\mathrm{P}_{11}-\mathrm{P}_{15}$ are shown in Figure 6 and Figure 7 . 


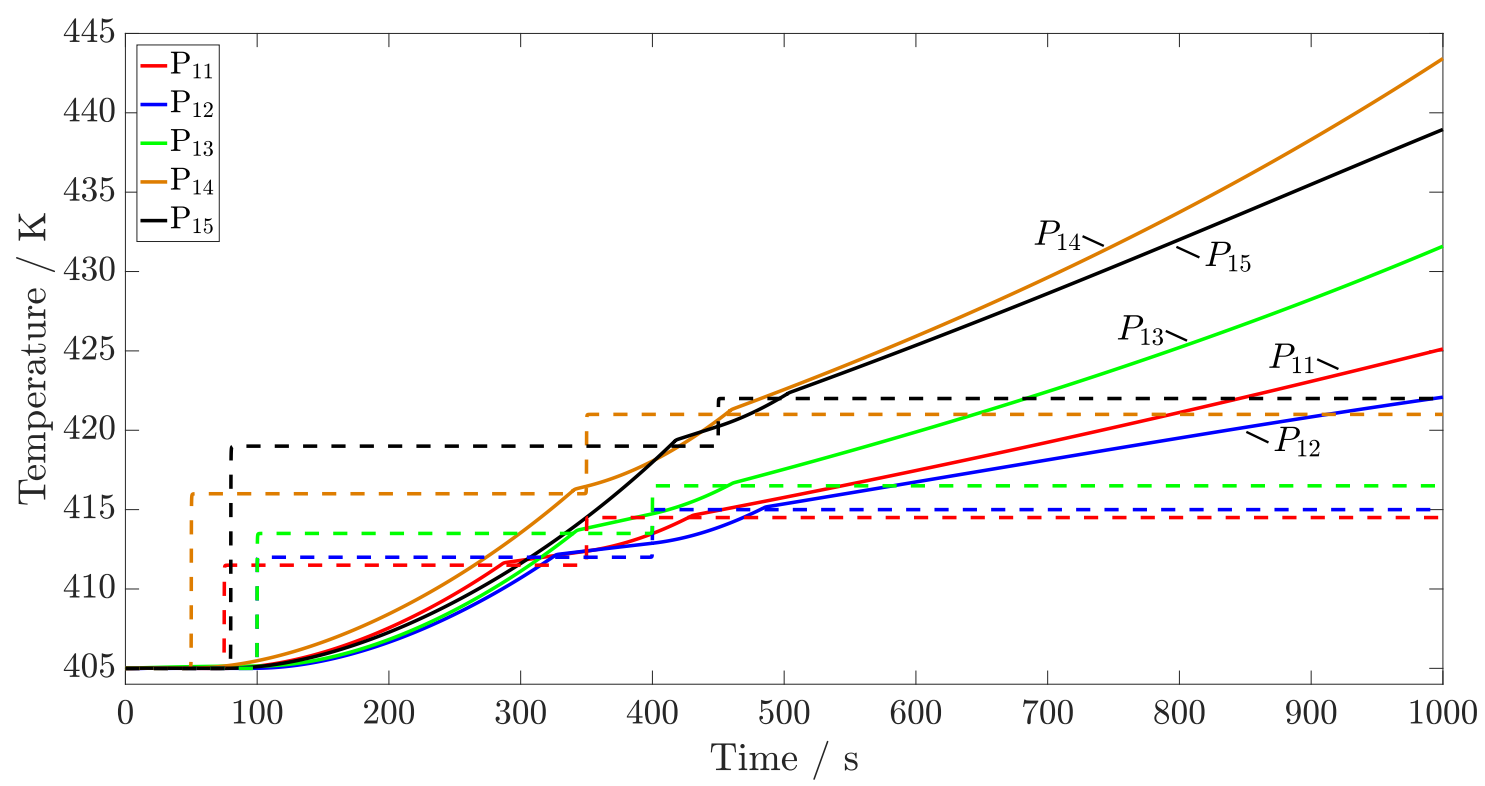

Figure 6: Temperature profiles for processes $\mathrm{P}_{11}-\mathrm{P}_{15}$.

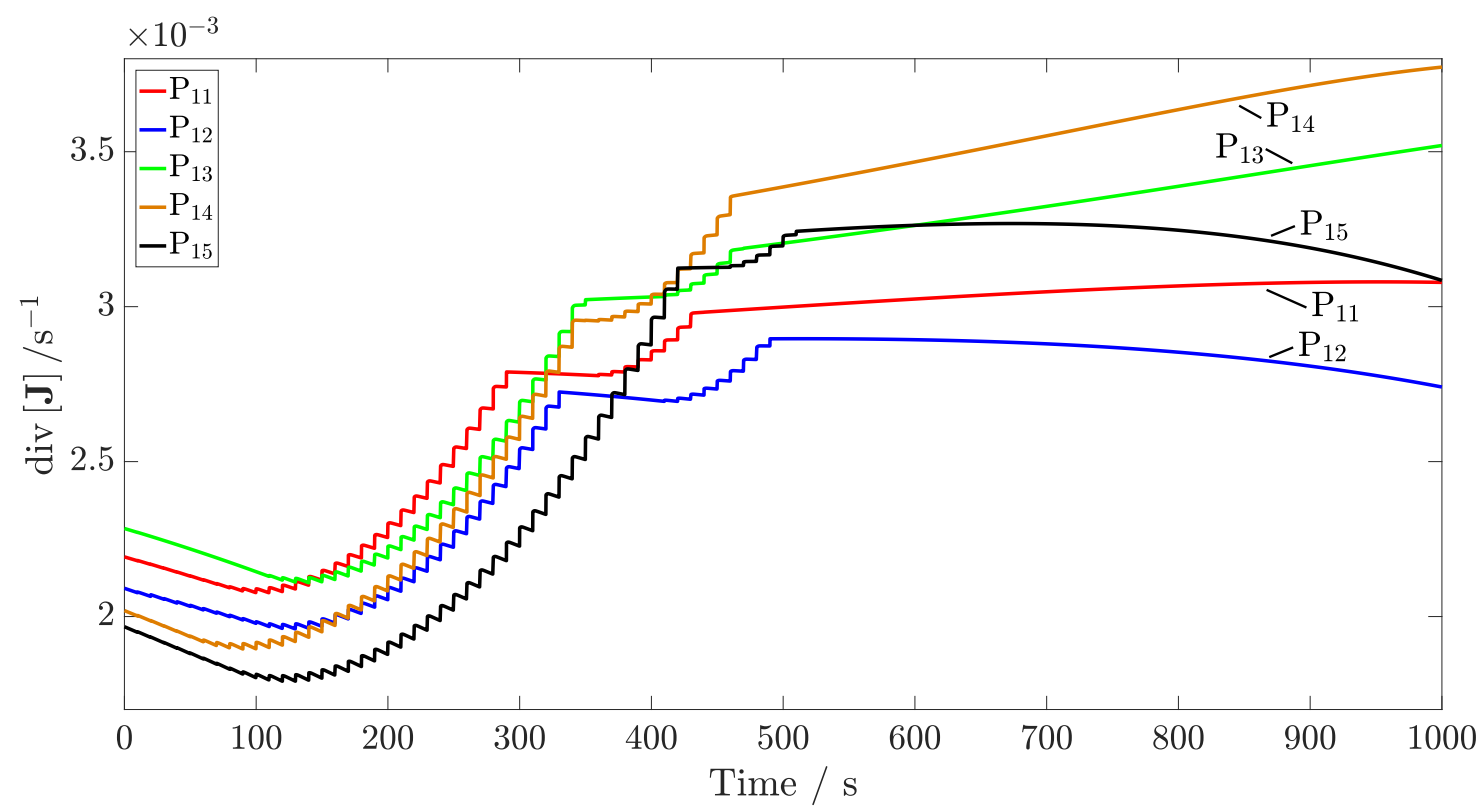

Figure 7: Divergence profiles for processes $\mathrm{P}_{11}-\mathrm{P}_{15}$.

For processes $\mathrm{P}_{11}, \mathrm{P}_{13}$ and $\mathrm{P}_{14}$ the same behaviour for $\operatorname{div}[\mathbf{J}]$ as for all other processes above is observed. During processes $\mathrm{P}_{12}$ and $\mathrm{P}_{15}$ the divergence decreases towards the end of the reaction are due to the fast kinetics which cause the concentration of each reactant to decrease very quickly, hence resulting in a stabilising effect for the overall system.

Similar trajectories for reactor temperature and divergence of the Jacobian are obtained for processes $\mathrm{P}_{11}-\mathrm{P}_{20}$. The key information obtained from these graphs is that the behaviour 
311 of $\operatorname{div}[\mathbf{J}]$ is in accordance with the temperature of the system at the boundary of stability.

312 The temperature and divergence profiles for processes $\mathrm{P}_{16}-\mathrm{P}_{20}$ are shown in Figure 8 and 313 Figure 9.

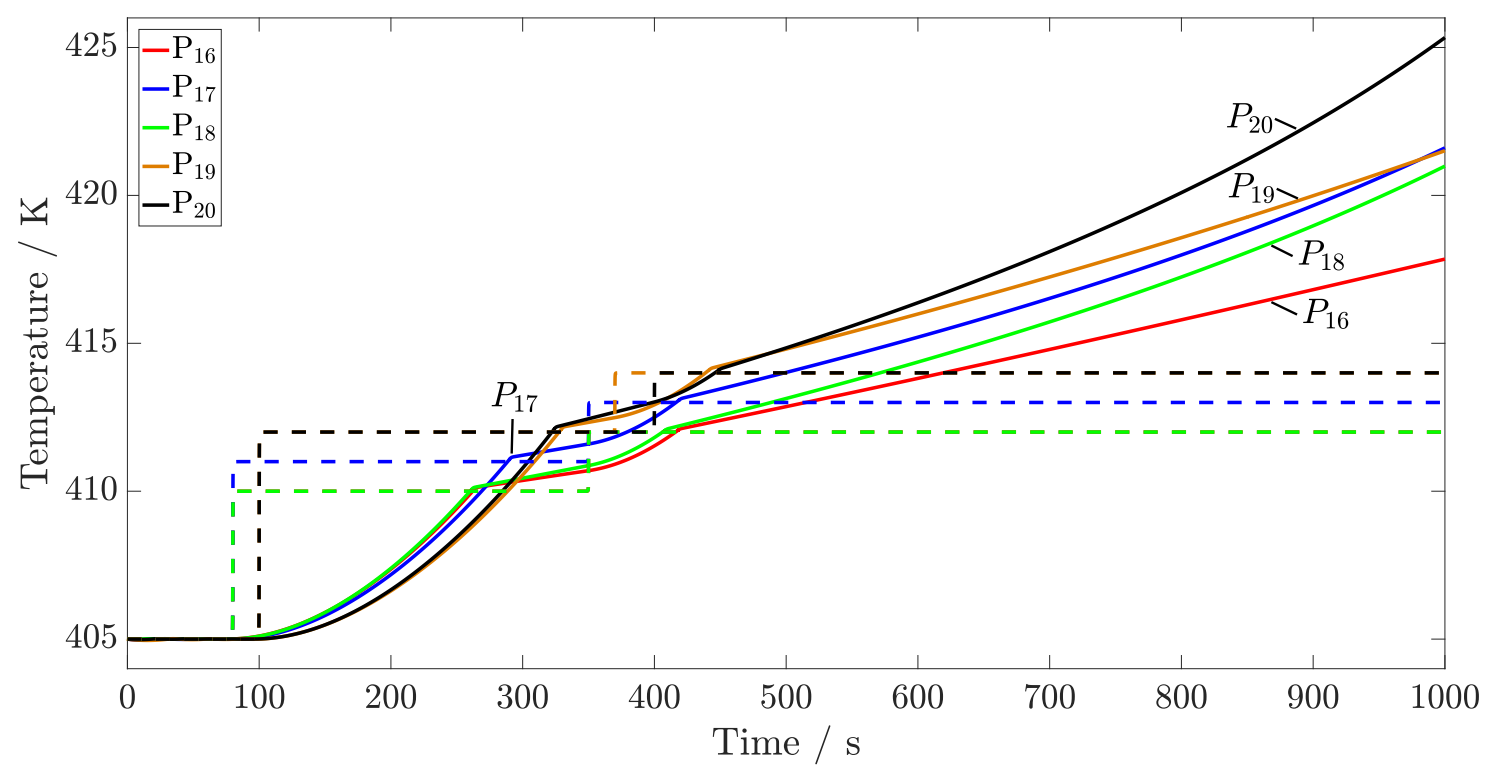

Figure 8: Temperature profiles for processes $\mathrm{P}_{16}-\mathrm{P}_{20}$.

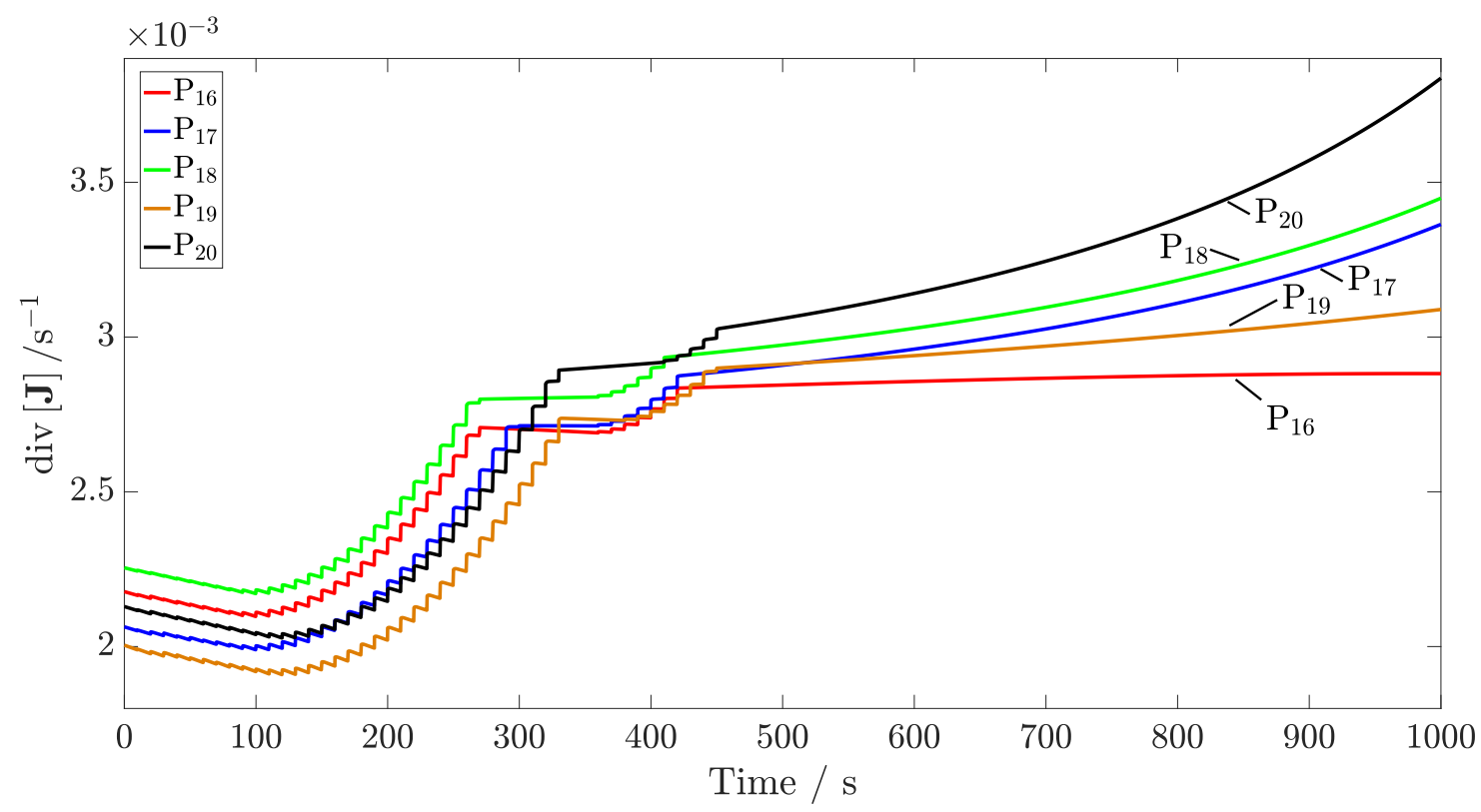

Figure 9: Divergence profiles for processes $\mathrm{P}_{16}-\mathrm{P}_{20}$.

From Figures 2 and 9 one common feature for $\operatorname{div}[\mathbf{J}]$ can be observed: even for stable operation of the batch processes the divergence is positive due to the constantly changing 
properties as no steady-state is present. The condition that $\operatorname{div}[\mathbf{J}] \leq 0$ during stable operation is therefore only valid if the temperature of the system is very low, rendering it infeasible for intensifying batch processes. To prove this condition, processes $\mathrm{P}_{16}-\mathrm{P}_{20}$ are simulated, but with much lower initial temperatures. The temperature and divergence profiles for these processes are shown in Figures 10 and 11.

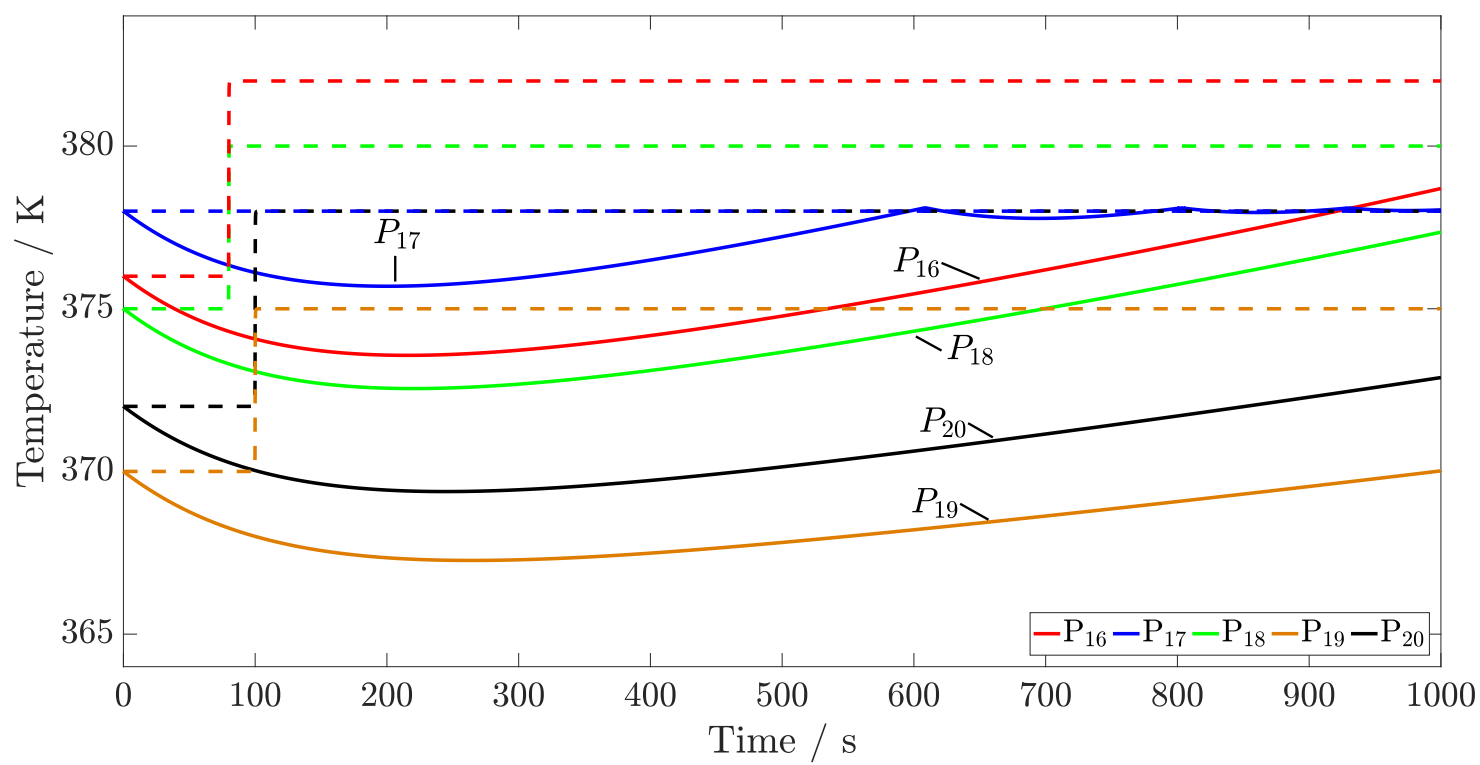

Figure 10: Temperature profiles for processes $\mathrm{P}_{16}-\mathrm{P}_{20}$.

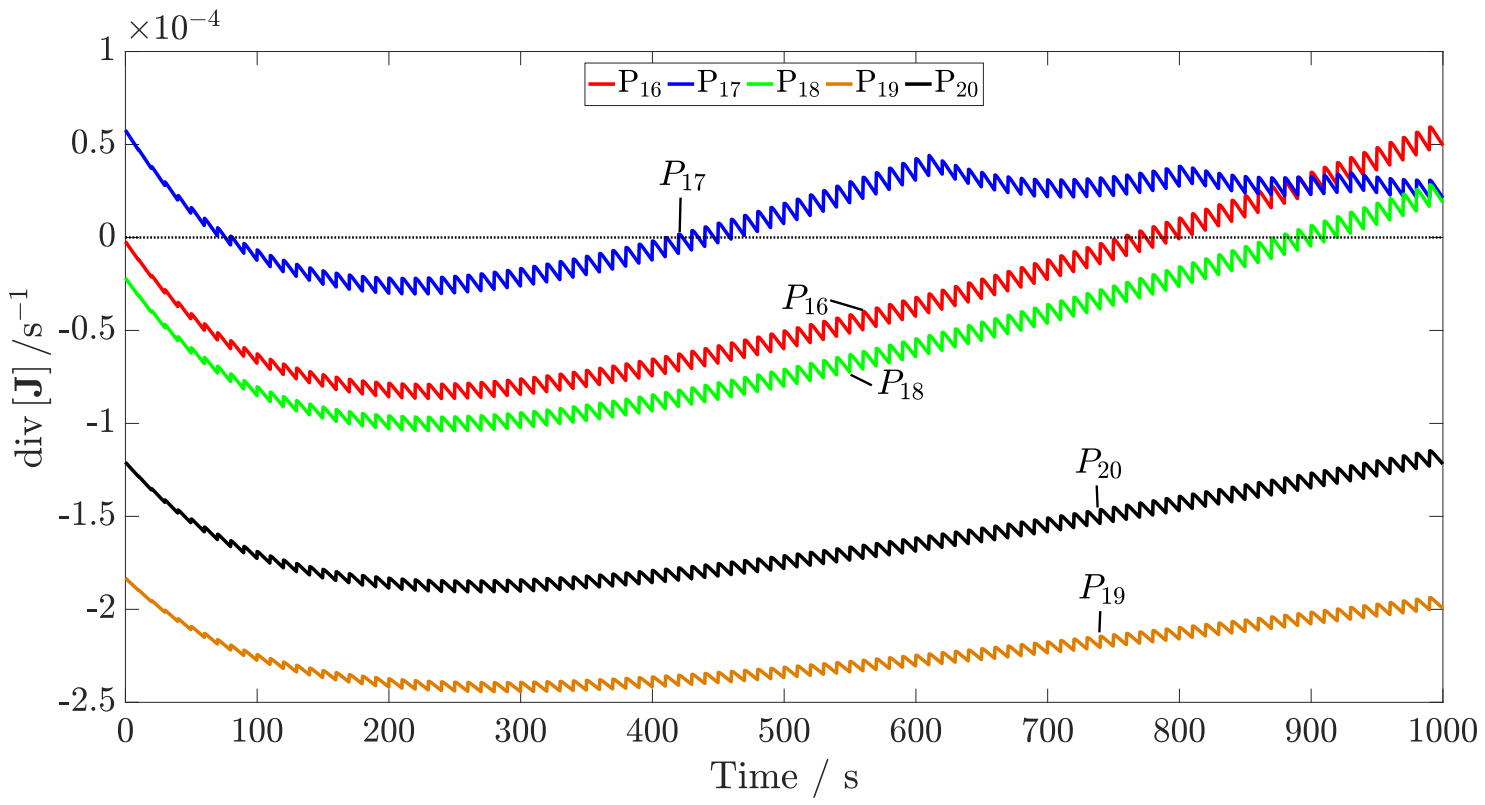

Figure 11: Divergence profiles for processes $\mathrm{P}_{16}-\mathrm{P}_{20}$. 
As can be seen in Figures 10 and 11, only for extremely low temperatures does the divergence criterion become negative. This significant deviation of the stability criterion $\operatorname{div}[\mathbf{J}] \leq 0$ to the actual boundary of stability hence requires a function to evaluate stability criterion $\mathcal{K}$, which was introduced in Kähm and Vassiliadis (2018).

\subsubsection{Sensitivity analysis of divergence criterion}

The values of the divergence criterion, as shown in Figures $3-10$, are of the order of $10^{-3}$. This is not due to numerical effects of the ODE solver employed, since the divergence is evaluated exactly using algebraic expressions (Equation (3.6)).

To prove this point, a sensitivity analysis of process $\mathrm{P}_{5}$ is carried out with varying tolerances for the ODE solver employed. The tolerances $\epsilon_{t o l}$ used, from lowest to highest accuracy, are $\epsilon_{t o l}=10^{-6}, 10^{-7}, 10^{-8}, 5 \times 10^{-9}, 4 \times 10^{-9}$. The simulation using the highest accuracy, namely $\epsilon_{t o l}=4 \times 10^{-9}$, is used as the reference. The error with respect to the reference trajectory is plotted on a logarithmic scale in Figure 12.

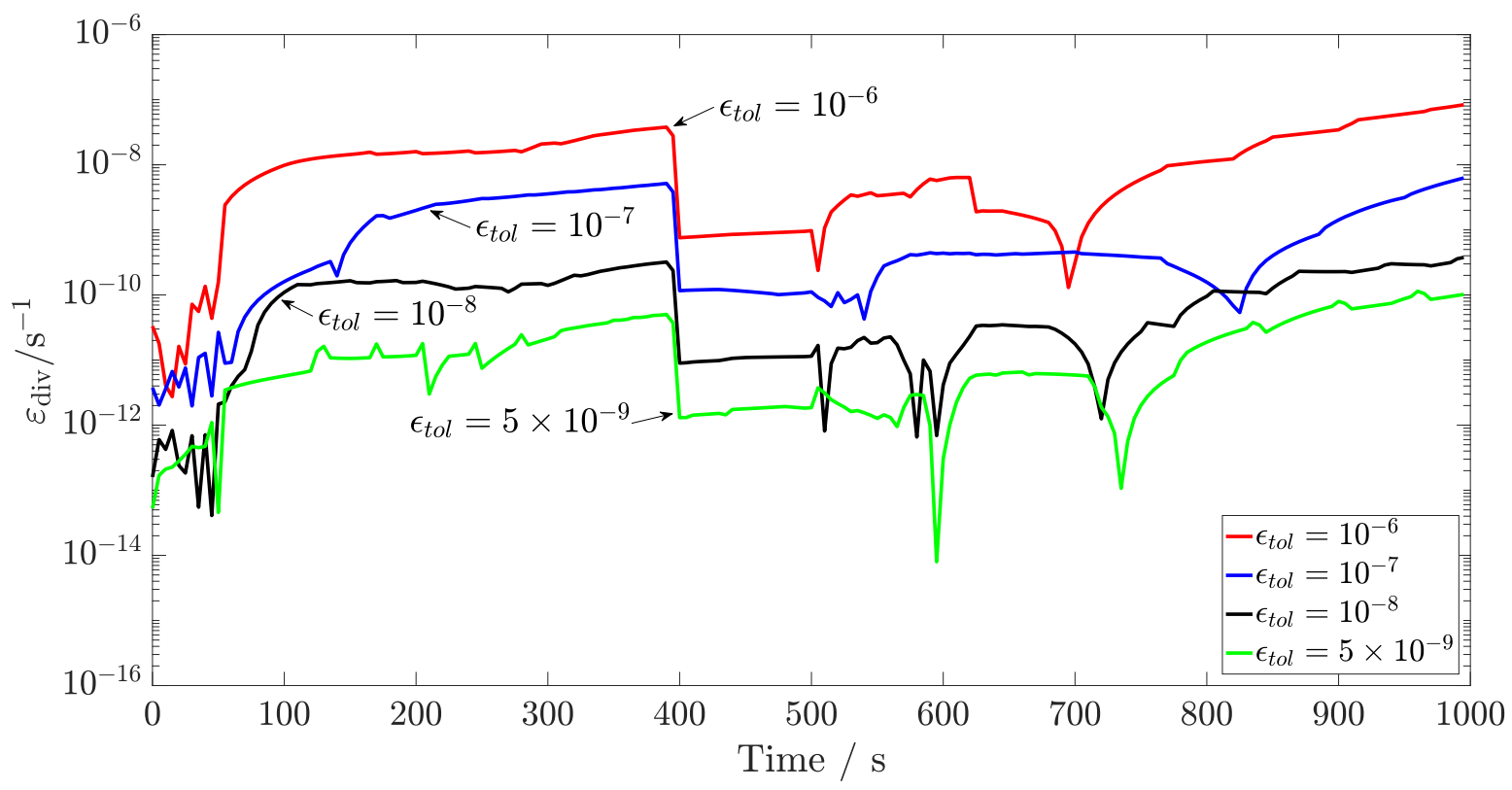

Figure 12: Logarithmic plot of error profiles for each sensitivity setting with respect to $\epsilon_{\text {tol }}=4 \times 10^{-9}$ for process $\mathrm{P}_{5}$.

As can be seen in Figure 12 the errors are below $10^{-6}$ at all times.

The tolerance setting used throughout all simulations is $\epsilon_{t o l}=10^{-8}$. As can be seen from Figure 12 the error for the divergence obtained is below $10^{-8}$ at all times. Hence, the numerical effects due to the ODE solver used do not cause the divergence to be positive during stable operation. 


\subsection{Results of the analysis}

The necessity of long prediction horizons to obtain reliable results for the Lyapunov exponent method limits its use when embedded in MPC. This is due to the increased computational time required to evaluate the thermal stability with Lyapunov exponents.

The divergence method, as was shown above for processes $\mathrm{P}_{1}-\mathrm{P}_{20}$, requires less computational time, but does not describe the transition to instability reliably. Only in batch processes with very low reactor temperatures does the divergence criterion indicate stable operation.

The divergence method results in a systematic offset of the loss of stability into the positive region for $\operatorname{div}[\mathbf{J}]$.

A stability criterion, for which the divergence of the Jacobian matrix is reduced by a correction function, can describe the point at which the loss of thermal stability for exothermic batch reactors occurs with little computational time.

\section{Stability criterion $\mathcal{K}$}

Stability criterion $\mathcal{K}$ describes the transition of thermal stability for exothermic batch processes. During stable operation the criterion $\mathcal{K}$ has to be smaller than or equal to zero:

$$
\mathcal{K} \leq 0 \rightarrow \text { stableprocess }
$$

When the process analysed becomes unstable due to a thermal runaway, the criterion becomes positive.

The stability criterion $\mathcal{K}$ is derived in the form of a difference equation, based on the divergence criterion of the Jacobian matrix div [J] (Strozzi and Zaldívar, 1999; Arnold, 1973) and a correction function $\mathcal{E}$ (Kähm and Vassiliadis, 2018). In the evaluation step $i$ at time $t^{(i)}$ the criterion $\mathcal{K}$ is evaluated by taking the difference between the divergence at step $i$, $\operatorname{div}[\mathbf{J}]^{(i)}$, and the correction function at step $i, \mathcal{E}^{(i)}$ :

$$
\mathcal{K}^{(i)}=\operatorname{div}[\mathbf{J}]^{(i)}-\left|\mathcal{E}^{(i)}\right|
$$

The correction function $\mathcal{E}^{(i)}$ is an approximation of $\operatorname{div}[\mathbf{J}]^{(i)}$ at the point of transition from stable to unstable operation. It is evaluated using $\operatorname{div}[\mathbf{J}]^{(i-1)}$ in the previous step $i-1$ and the dimensionless numbers which influence the divergence:

$$
\mathcal{E}^{(i)}=f\left(\operatorname{div}[\mathbf{J}]^{(i-1)}, D a^{(i)}, D a^{(i-1)}, B^{(i)}, B^{(i-1)}, S t^{(i)}, S t^{(i-1)}, \gamma^{(i)}, \gamma^{(i-1)}\right)
$$

where he dimensionless variables in Equation (4.3) are called the Barkelew number $B$, the Arrhenius number $\gamma$, the Damköhler number $D a$, and the Stanton number St. 
To further investigate stability criterion $\mathcal{K}$ a detailed analysis on the divergence is required, which is presented in the following section.

\subsection{Derivation}

From the expression found for the divergence, stability criterion $\mathcal{K}$ is derived. This is done by varying each individual variable at a time, while keeping the others fixed, until the resulting process is unstable. The gradient of the divergence with respect to that variable at the boundary of stability then becomes the particular gradient coefficient.

In order to derive the gradient coefficients, the effect of varying the reaction rate constant $k_{0}$, the enthalpy of reaction $\Delta H_{r}$, the heat transfer coefficient $U$ and the activation energy $E_{a}$ are considered. The correction function $\mathcal{E}$ is obtained after a first order Taylor expansion of the logarithm of the divergence, $\ln (\operatorname{div}[\mathbf{J}])$. The resulting expression is given by:

$$
\begin{aligned}
\mathrm{d} \ln (\operatorname{div}[\mathbf{J}]) & =\left(\frac{\partial \ln (\operatorname{div}[\mathbf{J}])}{\partial \ln (B)}\right)_{\frac{D a_{\text {res }}}{t_{\text {ref }}}, \gamma, \frac{S t}{t_{\text {ref }}}} \mathrm{d} \ln (B)+\left(\frac{\partial \ln (\operatorname{div}[\mathbf{J}])}{\partial \ln \left(\frac{D a_{\text {res }}}{t_{\text {ref }}}\right)}\right)_{B, \gamma, \frac{S t}{t_{\text {ref }}}} \mathrm{d} \ln \left(\frac{D a_{\text {res }}}{t_{\text {ref }}}\right) \\
& +\left(\frac{\partial \ln (\operatorname{div}[\mathbf{J}])}{\partial \ln (\gamma)}\right)_{B, \frac{D a_{\text {res }}}{t_{\text {ref }}}, \frac{S t}{t_{\text {ref }}}} \mathrm{d} \ln (\gamma)+\left(\frac{\partial \ln (\operatorname{div}[\mathbf{J}])}{\partial \ln \left(\frac{S t}{t_{\text {ref }}}\right)}\right)_{B, \frac{D a_{\text {res }}}{t_{\text {ref }}}, \gamma} \mathrm{d} \ln \left(\frac{S t}{t_{\text {ref }}}\right) \\
\mathrm{d} \ln (\operatorname{div}[\mathbf{J}]) & =m_{B} \mathrm{~d} \ln (B)+m_{D a_{\text {res }}} \mathrm{d} \ln \left(\frac{D a_{\text {res }}}{t_{\text {ref }}}\right)+m_{\gamma} \mathrm{d} \ln (\gamma)+m_{S t} \mathrm{~d} \ln \left(\frac{S t}{t_{\text {ref }}}\right)
\end{aligned}
$$

where $m$ are the gradient coefficients obtained from the Taylor expansion. A detailed derivation of this expression is given in Kähm and Vassiliadis (2018). The variable $D a_{\text {res }}$ is the resultant Damköhler number of the system which is discussed below. In Kähm and Vassiliadis (2018) gradient coefficients for a single reaction depending solely on component A were found, which are given in Table 5.

Table 5: Gradient coefficients found for a single reaction depending on one component (Kähm and Vassiliadis, 2018).

\begin{tabular}{ccccc}
\hline Gradient coefficient & $m_{B}$ & $m_{D a_{\text {res }}}$ & $m_{\gamma}$ & $m_{S t}$ \\
\hline Value & 1.28 & 1.21 & -26.9 & -0.187 \\
\hline
\end{tabular}

Using the derivation of the divergence, stability criterion $\mathcal{K}$ will now be derived for these reaction schemes. The dimensionless variables found in Equations (3.7a) - (3.7e) are used to find an expression for $\mathcal{E}^{(i)}$. As can be seen in Equation (3.6), two Damköhler numbers appear within the divergence of the batch system due to the presence of two reagents influencing the reaction rate. Therefore a weighted average of both Damköhler numbers is required for the derivation of the gradient coefficients for the function $\mathcal{E}^{(i)}$. From Equation (3.6) it can 
be seen that a natural form of the resultant Damköhler number $D a_{\text {res }}$ should be given by the following expression:

$$
D a_{\text {res }}^{(i)}=n_{\mathrm{A}} \nu_{\mathrm{A}} D a_{\mathrm{A}}^{(i)}+n_{\mathrm{B}} \nu_{\mathrm{B}} D a_{\mathrm{B}}^{(i)}
$$

To check the validity of this expression, the form of criterion $\mathcal{K}$ from Kähm and Vassiliadis (2018), where the reaction does not depend on the concentration of reactant B, is obtained by setting $n_{B}=0$ in Equations $(3.7 c)-(3.7 d)$. This reduces $D a_{\text {res }}$ to give $D a_{\text {res }}=n_{\mathrm{A}} \nu_{\mathrm{A}} D a_{\mathrm{A}}$, which is the expression found in Kähm and Vassiliadis (2018). Hence the form of $D a_{\text {res }}$ presented above is logical and will be used throughout this work. Furthermore, the influence of stoichiometric coeffifients and reaction orders with respect to each reagent present for each reaction is hence included in this manner.

$$
\begin{aligned}
\mathcal{E}^{(i)}= & \operatorname{div}[\mathbf{J}]^{(i-1)}\left[1+m_{S t} \ln \left(\frac{S t^{(i)}}{S t^{(i-1)}}\right)+m_{B} \ln \left(\frac{B^{(i)}}{B^{(i-1)}}\right)\right. \\
& \left.+m_{D a_{\mathrm{res}}} \ln \left(\frac{n_{\mathrm{A}} \nu_{\mathrm{A}} D a_{\mathrm{A}}^{(i)}+n_{\mathrm{B}} \nu_{\mathrm{B}} D a_{\mathrm{B}}^{(i)}}{n_{\mathrm{A}} \nu_{\mathrm{A}} D a_{\mathrm{A}}^{(i-1)}+n_{\mathrm{B}} \nu_{\mathrm{B}} D a_{\mathrm{B}}^{(i-1)}}\right)+m_{\gamma} \ln \left(\frac{\gamma^{(i)}}{\gamma^{(i-1)}}\right)\right]
\end{aligned}
$$

Using the following definition of the derivative of a logarithm:

$$
\mathrm{d} \ln (y)=\frac{\mathrm{d} y}{y}=\lim _{\Delta y^{i} \rightarrow 0} \frac{\Delta y^{(i)}}{y^{(i-1)}} \approx \frac{y^{(i)}-y^{(i-1)}}{y^{(i-1)}}
$$

an expression for the stability criterion $\mathcal{K}$ at step $i$, using the definition in Equation (4.2), can be found:

$$
\begin{aligned}
\mathcal{K}^{(i)}= & \operatorname{div}[\mathbf{J}]^{(i)}-\left|\mathcal{E}^{(i)}\right| \\
\mathcal{K}^{(i)}= & \operatorname{div}[\mathbf{J}]^{(i)}-\mid \operatorname{div}[\mathbf{J}]^{(i-1)}\left[1+m_{S t} \frac{S t^{(i)}-S t^{(i-1)}}{S t^{(i-1)}}+m_{B} \frac{B^{(i)}-B^{(i-1)}}{B^{(i-1)}}\right. \\
& +m_{D a_{\mathrm{res}}} \frac{\left(n_{\mathrm{A}} \nu_{\mathrm{A}} D a_{\mathrm{A}}^{(i)}+n_{\mathrm{B}} \nu_{\mathrm{B}} D a_{\mathrm{B}}^{(i)}\right)-\left(n_{\mathrm{A}} \nu_{\mathrm{A}} D a_{\mathrm{A}}^{(i-1)}+n_{\mathrm{B}} \nu_{\mathrm{B}} D a_{\mathrm{B}}^{(i-1)}\right)}{n_{\mathrm{A}} \nu_{\mathrm{A}} D a_{\mathrm{A}}^{(i-1)}+n_{\mathrm{B}} \nu_{\mathrm{B}} D a_{\mathrm{B}}^{(i-1)}} \\
& \left.+m_{\gamma} \frac{\gamma^{(i)}-\gamma^{(i-1)}}{\gamma^{(i-1)}}\right] \mid
\end{aligned}
$$

This is a similar expression as given in Kähm and Vassiliadis (2018), with the following difference: the reaction rate depends on both reagents $\mathrm{A}$ and $\mathrm{B}$. Hence, both components appear in the heat generation term in Equation (3.4). This means that both A and B contribute 
towards the expression of the divergence obtained. Therefore two Damköhler numbers are obtained, given by Equations (3.7c) and (3.7d). Both Damköhler numbers depend on the pre-exponential Arrhenius factor $k_{0}$ and the concentrations $[\mathrm{A}]$ and [B]. This dependence on each other leads to the problem that it is not possible to vary each dimensionless number without varying the other. Therefore, a weighted sum of both as shown in Equation (4.6) is tested for the derivation of criterion $\mathcal{K}$ leading to the following expression:

$$
\begin{aligned}
\mathcal{K}^{(i)}= & \operatorname{div}[\mathbf{J}]^{(i)}-\mid \operatorname{div}[\mathbf{J}]^{(i-1)}\left[1+m_{S t} \frac{S t^{(i)}-S t^{(i-1)}}{S t^{(i-1)}}\right. \\
& \left.+m_{B} \frac{B^{(i)}-B^{(i-1)}}{B^{(i-1)}}+m_{D a_{\mathrm{res}}} \frac{D a_{\mathrm{res}}^{(i)}-D a_{\mathrm{res}}^{(i-1)}}{D a_{\mathrm{res}}^{(i-1)}}+m_{\gamma} \frac{\gamma^{(i)}-\gamma^{(i-1)}}{\gamma^{(i-1)}}\right] \mid
\end{aligned}
$$

This is the final expression for $\mathcal{K}$ used for the PI-control and MPC scheme implementations.

As the value of gradients $m_{D a_{\text {res }}}, m_{B}, m_{\gamma}$ and $m_{S t}$ decreases, the value of $\mathcal{E}$, which estimates the divergence at the boundary of stability, decreases. According to Equation (4.9), as the value of $\mathcal{E}$ is smaller, the condition of $\mathcal{K}$ becoming positive and hence predicting instability occurs earlier, making it a conservative stability measure. Therefore, from all the values for the gradients coefficients found, the most conservative ones are used for the function of $\mathcal{E}$.

In the following sections the derivation of gradient coefficients $m_{D a_{\text {res }}}, m_{B}, m_{\gamma}$ and $m_{S t}$ are carried out.

\subsection{Determination of gradient coefficients}

\section{Variation with respect to Damköhler number}

The gradient at the boundary of stability with respect to the Damköhler number, $D a_{\text {res }}$, is analysed first. To combine the influence of both reactants $\mathrm{A}$ and $\mathrm{B}, D a_{\mathrm{res}}$ is given by Equation (4.6). In contrast to the base processes $\mathrm{P}_{1}-\mathrm{P}_{20}$ in this section the pre-exponential Arrhenius coefficient $k_{0}$ is increased until loss of stability, hence giving processes denoted by $\mathrm{P}_{1,(D a)}-\mathrm{P}_{20,(D a)}$. A thermal runaway is caused by increasing the rate of reaction until the heat generated by the reaction exceeds the cooling capacity. All remaining parameters of each process are kept constant. The processes obtained when varying the other dimensionless variables is denoted in a similar fashion.

The plots for processes $\mathrm{P}_{1,(D a)}-\mathrm{P}_{5,(D a)}$ are given in Figure 13. 


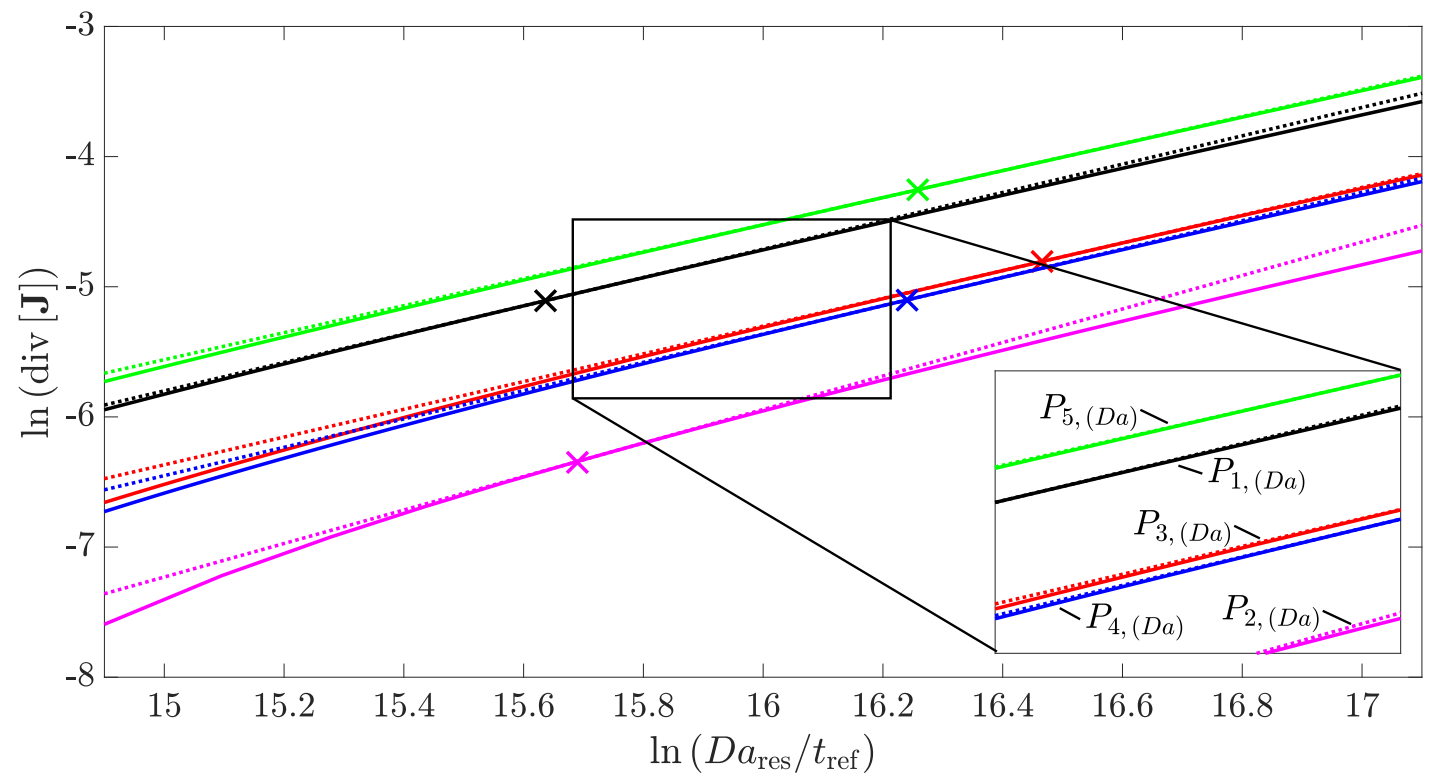

Figure 13: Variation of the divergence with respect to $D a_{\text {res }} / t_{\text {ref }}$ for processes $\mathrm{P}_{1,(D a)}-\mathrm{P}_{5,(D a)}$. The crosses indicate the points at the boundary of instability, and the dashed lines indicate the gradient at these points.

At the point where systems $\mathrm{P}_{1,(D a)}-\mathrm{P}_{20,(D a)}$ become unstable the value of $D a_{\text {res }}$ is recorded and the gradient of $\ln (\operatorname{div}[\mathbf{J}])$ at that point is found. This is indicated in Figure 13 as dashed lines. As can be seen, the lines are close to parallel. Therefore, the most conservative gradient obtained will give a good description of the divergence at the boundary of stability. Similar profiles and gradients are obtained for processes $\mathrm{P}_{6,(D a)}-\mathrm{P}_{20,(D a)}$, as will be shown below.

The extension of the Damköhler number for multi-component reactions is not trivial, as it is important to consider how much the reaction order and stoichiometric coefficient influence the resultant Damköhler number which can then be implemented for criterion $\mathcal{K}$. Since from the divergence the form given in Equation (4.6) is present, additional verification of the resulting gradient coefficients obtained at the boundary of instability are required. This thorough verification is required as the extension for multi-component reaction for criterion $\mathcal{K}$ has not been carried out before.

Therefore 5 more processes for which only the reaction orders and initial reaction temperatures are varied will be tested. The process parameters for processes $\mathrm{P}_{21}-\mathrm{P}_{25}$ are shown in Table 6. 
Table 6: Process parameters varied for processes $\mathrm{P}_{21}-\mathrm{P}_{25}$.

\begin{tabular}{cccc}
\hline Process & $n_{\mathrm{A}}$ & $n_{\mathrm{B}}$ & $T_{R 0}$ \\
& {$[-]$} & {$[-]$} & {$[\mathrm{K}]$} \\
& & & \\
\hline $\mathrm{P}_{21}$ & 2.5 & 1.0 & 420 \\
$\mathrm{P}_{22}$ & 3.5 & 1.0 & 390 \\
$\mathrm{P}_{23}$ & 3.5 & 1.5 & 370 \\
$\mathrm{P}_{24}$ & 3.5 & 2.5 & 335 \\
$\mathrm{P}_{25}$ & 3.5 & 3.0 & 310 \\
\hline
\end{tabular}

446 The same reactor parameters as for processes $\mathrm{P}_{16}-\mathrm{P}_{20}$ in Table 3 are used for processes ${ }_{447} \mathrm{P}_{21}-\mathrm{P}_{25}$.

The following parameters are held constant throughout processes $\mathrm{P}_{21}-\mathrm{P}_{25}$ :

$$
\begin{aligned}
k_{0} & =5.00 \times 10^{3} \frac{\mathrm{m}^{3(n-1)}}{\mathrm{kmol}^{(n-1)} \mathrm{s}} \\
\Delta H_{r} & =-180 \frac{\mathrm{kJ}}{\mathrm{mol}} \\
\nu_{\mathrm{A}} & =1.5 \\
\nu_{\mathrm{B}} & =2.5 \\
E_{a} / R & =9525 \mathrm{~K}
\end{aligned}
$$

448 where $n=n_{A}+n_{B}$.

449 If for these processes approximately parallel gradients are obtained as well, then this form 450 of the resultant Damköhler number can be used for multi-component reactions. The gradient 451 plots obtained for processes $\mathrm{P}_{21}-\mathrm{P}_{25}$ are shown in Figure 14 . 


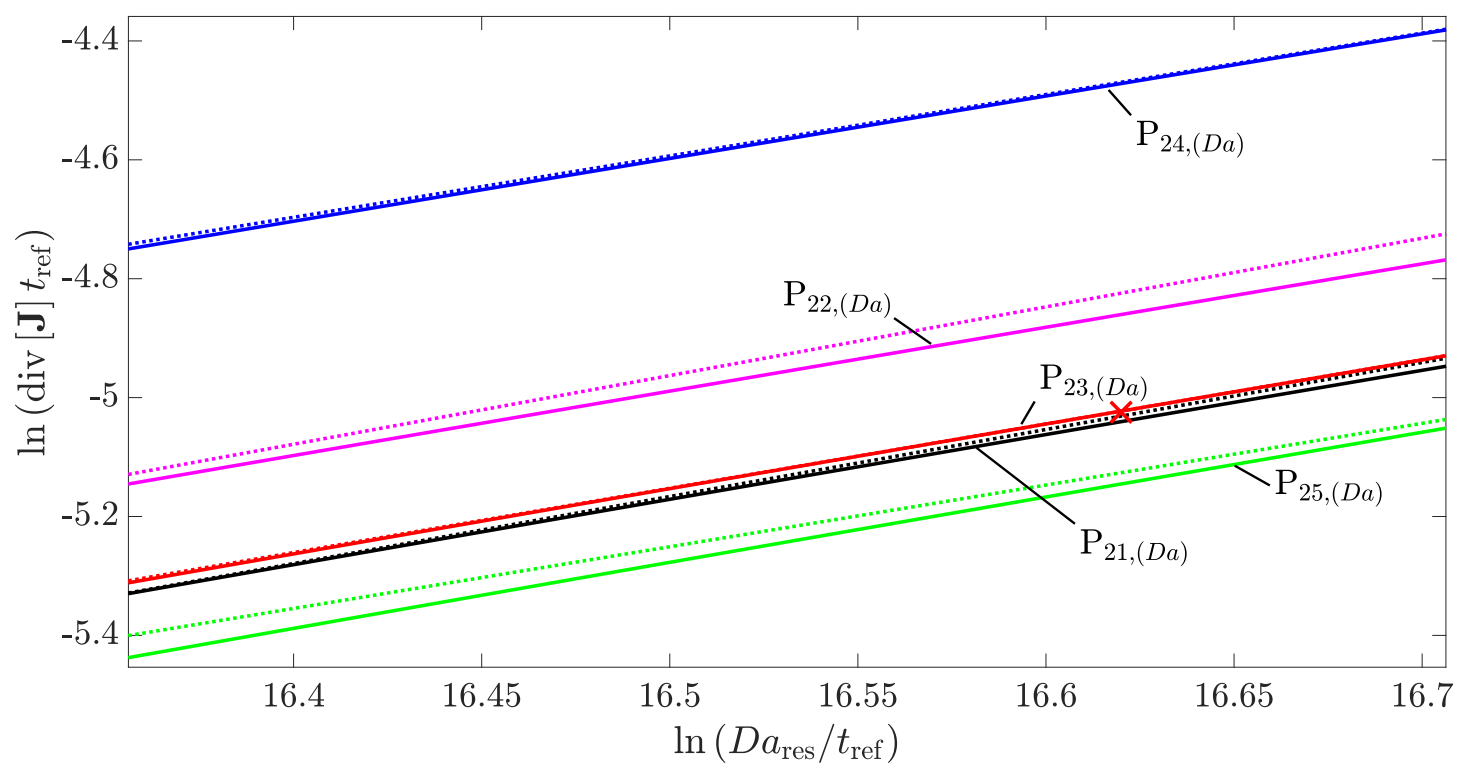

Figure 14: Variation of the divergence with respect to $D a_{\text {res }} / t_{\text {ref }}$ for processes $\mathrm{P}_{21,(D a)}-\mathrm{P}_{25,(D a)}$. The crosses indicate the points at the boundary of instability, and the dashed lines indicate the gradient at these points.

It is seen from Figure 14 that the resulting gradient lines for strongly varying reaction orders are still parallel. Therefore it is demonstrated that the form of the resultant Damköhler number shown in Equation (4.6) is valid for multi-component reactions. Hence gradient coefficients for this resultant Damköhler number can be used to quantify the divergence at the boundary of stability.

The evaluated gradient coefficients $m_{D a_{\mathrm{res}}}$ for $\ln (\operatorname{div}[\mathbf{J}])$ with respect to $\ln \left(D a_{\mathrm{res}} / t_{\mathrm{ref}}\right)$ at the boundary of stability for processes $\mathrm{P}_{1,(D a)}-\mathrm{P}_{20,(D a)}$ are shown in Table 7.

Table 7: Gradient coefficient $m_{D a}$ values for processes $\mathrm{P}_{1,(D a)}-\mathrm{P}_{25,(D a)}$.

\begin{tabular}{ccccccccccc}
\hline Process & $\mathrm{P}_{1}$ & $\mathrm{P}_{2}$ & $\mathrm{P}_{3}$ & $\mathrm{P}_{4}$ & $\mathrm{P}_{5}$ & $\mathrm{P}_{6}$ & $\mathrm{P}_{7}$ & $\mathrm{P}_{8}$ & $\mathrm{P}_{9}$ & $\mathrm{P}_{10}$ \\
$m_{D a_{\text {res }}}$ & 1.09 & 1.13 & 1.05 & 1.04 & 1.04 & 1.11 & 1.11 & 1.08 & 1.13 & 1.13 \\
\hline Process & $\mathrm{P}_{11}$ & $\mathrm{P}_{12}$ & $\mathrm{P}_{13}$ & $\mathrm{P}_{14}$ & $\mathrm{P}_{15}$ & $\mathrm{P}_{16}$ & $\mathrm{P}_{17}$ & $\mathrm{P}_{18}$ & $\mathrm{P}_{19}$ & $\mathrm{P}_{20}$ \\
$m_{D a_{\text {res }}}$ & 1.13 & 1.16 & 1.08 & 1.03 & 1.04 & 1.15 & 1.09 & 1.16 & 1.11 & 1.09 \\
\hline Process & $\mathrm{P}_{21}$ & $\mathrm{P}_{22}$ & $\mathrm{P}_{23}$ & $\mathrm{P}_{24}$ & $\mathrm{P}_{25}$ & & & & & \\
$m_{D a_{\text {res }}}$ & 1.25 & 1.17 & 1.06 & 1.05 & 1.04 & & & & \\
\hline
\end{tabular}

Variation with respect to Barkelew number

For the dependence on the divergence of the Jacobian matrix with respect to the Barkelew number $B$ the same logic is applied as for the Damköhler number. In processes $\mathrm{P}_{1,(B)}-\mathrm{P}_{20,(B)}$ 
the enthalpy of reaction $\Delta H_{r}$ is varied until instability occurs and a thermal runaway occurs. All remaining parameters are kept constant during this analysis. The value of $\ln (\operatorname{div}[\mathbf{J}])$ with respect to $\ln (B)$ for processes $\mathrm{P}_{1,(B)}-\mathrm{P}_{5,(B)}$ are given in Figure 15 .

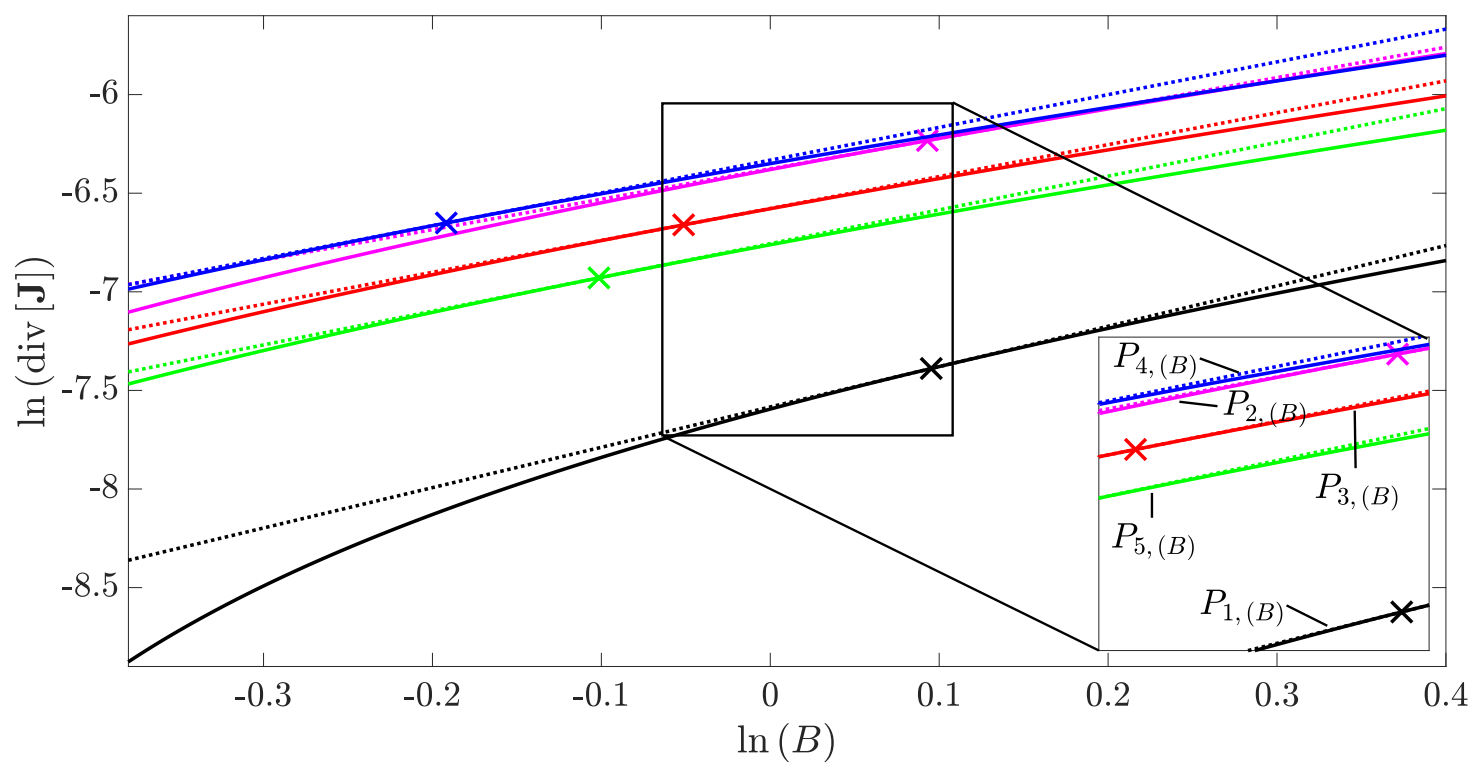

Figure 15: Variation of the divergence with respect to $B$ for processes $\mathrm{P}_{1,(B)}-\mathrm{P}_{5,(B)}$. The crosses indicate the points at the boundary of instability, and the dashed lines indicate the gradient at these points.

As for the Damköhler number, the lines obtained for processes $\mathrm{P}_{1,(B)}-\mathrm{P}_{5,(B)}$ at the boundary of stability are nearly parallel. The same behaviour is observed for processes $\mathrm{P}_{6,(B)}-\mathrm{P}_{20,(B)}$. The results obtained for $m_{B}$ are consistent with the values given in Kähm and Vassiliadis (2018). The values of $m_{B}$ for this reaction scheme are given in Table 8 .

Table 8: Gradient coefficient $m_{B}$ values for processes $\mathrm{P}_{1,(B)}-\mathrm{P}_{20,(B)}$.

\begin{tabular}{ccccccccccc}
\hline Process & $\mathrm{P}_{1}$ & $\mathrm{P}_{2}$ & $\mathrm{P}_{3}$ & $\mathrm{P}_{4}$ & $\mathrm{P}_{5}$ & $\mathrm{P}_{6}$ & $\mathrm{P}_{7}$ & $\mathrm{P}_{8}$ & $\mathrm{P}_{9}$ & $\mathrm{P}_{10}$ \\
$m_{B}$ & 2.05 & 1.54 & 1.62 & 1.66 & 1.71 & 1.55 & 1.55 & 1.67 & 1.60 & 1.72 \\
\hline Process & $\mathrm{P}_{11}$ & $\mathrm{P}_{12}$ & $\mathrm{P}_{13}$ & $\mathrm{P}_{14}$ & $\mathrm{P}_{15}$ & $\mathrm{P}_{16}$ & $\mathrm{P}_{17}$ & $\mathrm{P}_{18}$ & $\mathrm{P}_{19}$ & $\mathrm{P}_{20}$ \\
$m_{B}$ & 1.57 & 1.47 & 1.53 & 1.58 & 1.80 & 1.34 & 1.30 & 1.35 & 1.28 & 1.34 \\
\hline
\end{tabular}

In order to get a conservative estimate of the divergence value at the boundary of stability, the most conservative gradient value from the ones found in Table 8 is used, i.e. specifically $m_{B}=1.28$. 
Variation with respect to Arrhenius number

The processes $\mathrm{P}_{1,(\gamma)}-\mathrm{P}_{20,(\gamma)}$ are based on the parameters for processes $\mathrm{P}_{1}-\mathrm{P}_{20}$ given in Table 1 . The reduction in activation energy $E_{a}$ increases the reaction rate, hence resulting in more heat generation. Once the heat generated exceeds the cooling capacity of the system and a thermal runaway occurs, the respective value of $E_{a}$ is recorded. All remaining parameters are kept constant during this analysis. The variation of $\operatorname{div}[\mathbf{J}]$ with respect to the Arrhenius number $\gamma$ for processes $\mathrm{P}_{1,(\gamma)}-\mathrm{P}_{5,(\gamma)}$ is given in Figure 16.

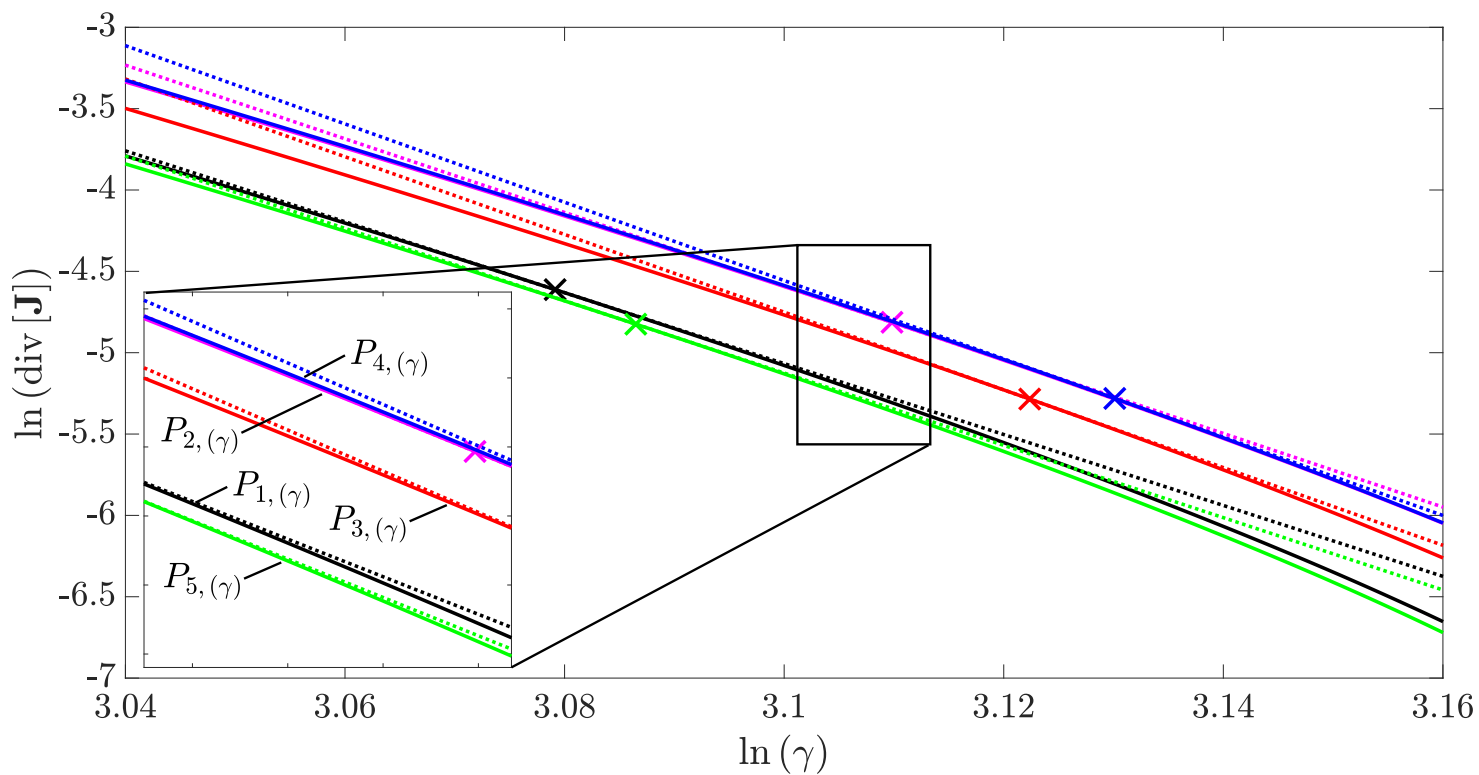

Figure 16: Variation of the divergence with respect to $\gamma$ for processes $\mathrm{P}_{1,(\gamma)}^{1}-\mathrm{P}_{5,(\gamma)}^{1}$. The crosses indicate the points at the boundary of instability, and the dashed lines indicate the gradient at these points.

As was observed above, the gradients obtained at the boundary of stability are very similar for all processes $\mathrm{P}_{1,(\gamma)}-\mathrm{P}_{20,(\gamma)}$. The values of $m_{\gamma}$ for this reaction scheme are given in Table 9.

Table 9: Gradient coefficient $m_{\gamma}$ values for processes $\mathrm{P}_{1,(\gamma)}-\mathrm{P}_{20,(\gamma)}^{1}$.

\begin{tabular}{ccccccccccc}
\hline Process & $\mathrm{P}_{1}$ & $\mathrm{P}_{2}$ & $\mathrm{P}_{3}$ & $\mathrm{P}_{4}$ & $\mathrm{P}_{5}$ & $\mathrm{P}_{6}$ & $\mathrm{P}_{7}$ & $\mathrm{P}_{8}$ & $\mathrm{P}_{9}$ & $\mathrm{P}_{10}^{1}$ \\
$m_{\gamma}$ & -21.8 & -22.6 & -23.9 & -24.1 & -22.2 & -23.7 & -23.8 & -24.7 & -24.7 & -23.1 \\
\hline Process & $\mathrm{P}_{11}$ & $\mathrm{P}_{12}$ & $\mathrm{P}_{13}$ & $\mathrm{P}_{14}$ & $\mathrm{P}_{15}$ & $\mathrm{P}_{16}$ & $\mathrm{P}_{17}$ & $\mathrm{P}_{18}^{1}$ & $\mathrm{P}_{19}$ & $\mathrm{P}_{20}$ \\
$m_{\gamma}$ & -22.4 & -23.3 & -22.5 & -23.8 & -22.2 & -22.3 & -23.05 & -24.5 & -24.3 & -23.3 \\
\hline
\end{tabular}

The most conservative value obtained from these processes is used in order to predict the value of the divergence close to the boundary of instability, i.e. specifically $m_{\gamma}=-21.8$. 
Variation with respect to Stanton number

The variation of $\operatorname{div}[\mathbf{J}]$ with respect to the Stanton number $S t$ for processes $\mathrm{P}_{1,(S t)}-\mathrm{P}_{20,(S t)}$ is analysed by varying the heat transfer coefficient $U$ with respect to the parameters of processes $\mathrm{P}_{1}-\mathrm{P}_{20}$. The lines obtained for $\operatorname{div}[\mathbf{J}]$ with respect to processes $\mathrm{P}_{1,(S t)}-\mathrm{P}_{5,(S t)}$ are given in Figure 17.

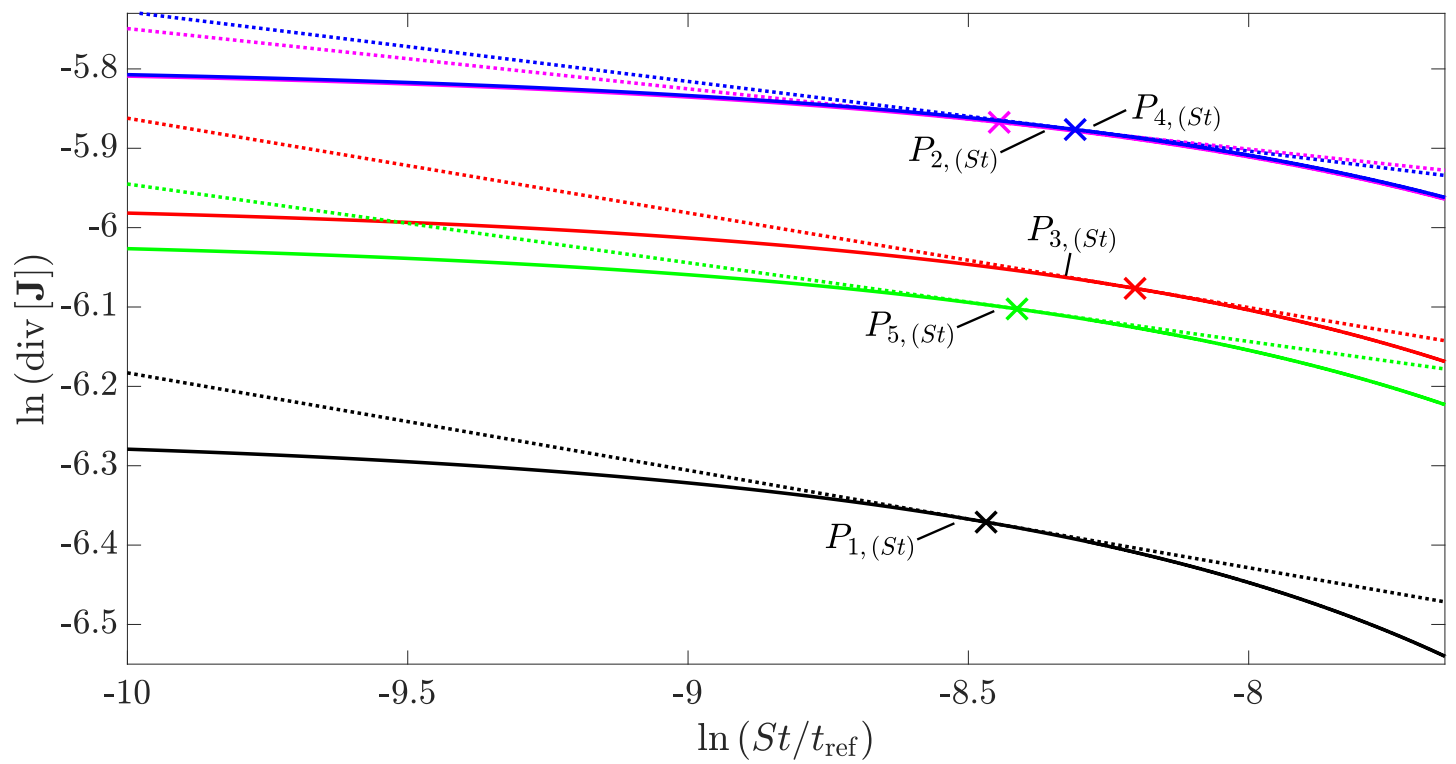

Figure 17: Variation of the divergence with respect to $S t / t_{\text {ref }}$ for processes $\mathrm{P}_{1,(S t)}-\mathrm{P}_{5,(S t)}$. The crosses indicate the points at the boundary of instability, and the dashed lines indicate the gradient at these points.

The gradients obtained for $\ln (\operatorname{div}[\mathbf{J}])$ at the boundary of stability were very close to each other. This is also the case for processes $\mathrm{P}_{1,(S t)}-\mathrm{P}_{20,(S t)}$. The values obtained for the gradient coefficients are shown in Table 10.

Table 10: Gradient coefficient $m_{\gamma}$ values for processes $\mathrm{P}_{1,(S t)}-\mathrm{P}_{20,(S t)}$.

\begin{tabular}{ccccccccccc}
\hline Process & $\mathrm{P}_{1}$ & $\mathrm{P}_{2}$ & $\mathrm{P}_{3}$ & $\mathrm{P}_{4}$ & $\mathrm{P}_{5}$ & $\mathrm{P}_{6}$ & $\mathrm{P}_{7}$ & $\mathrm{P}_{8}$ & $\mathrm{P}_{9}$ & $\mathrm{P}_{10}$ \\
$m_{S t}$ & -0.183 & -0.176 & -0.190 & -0.199 & -0.199 & -0.182 & -0.186 & -0.185 & -0.189 & -0.174 \\
\hline Process & $\mathrm{P}_{11}$ & $\mathrm{P}_{12}$ & $\mathrm{P}_{13}$ & $\mathrm{P}_{14}$ & $\mathrm{P}_{15}$ & $\mathrm{P}_{16}$ & $\mathrm{P}_{17}$ & $\mathrm{P}_{18}$ & $\mathrm{P}_{19}$ & $\mathrm{P}_{20}$ \\
$m_{S t}$ & -0.189 & -0.183 & -0.186 & -0.194 & -0.189 & -0.181 & -0.175 & -0.197 & -0.191 & -0.174 \\
\hline
\end{tabular}

From Table 10 the most conservative gradient obtained can therefore be used to predict the value of the divergence as the system comes closer to the boundary of stability, i.e. specifically $m_{S t}=-0.174$. 
The most conservative gradient coefficients are guaranteed to ensure stability. Hence the smallest values in magnitude from Tables $7-10$ are chosen to be used for function $\mathcal{K}$. The gradient coefficients used for all following simulations are given in Table 11.

Table 11: Most conservative gradient coefficients used for simulations.

\begin{tabular}{ccccc}
\hline Gradient coefficient & $m_{B}$ & $m_{D a_{\text {res }}}$ & $m_{\gamma}$ & $m_{S t}$ \\
\hline Value & 1.28 & 1.16 & -21.8 & -0.174 \\
\hline
\end{tabular}

The results obtained for all gradient coefficients are in accord with the results obtained in Kähm and Vassiliadis (2018). Small deviations in the values for $m_{D a_{\text {res }}}$ and $m_{S t}$ are present. Both deviations result in a more conservative prediction of instability by criterion $\mathcal{K}$. A significant deviation of the value for $m_{\gamma}$ is observed, which also results in a more conservative stability detection. The same value for $m_{B}$ is obtained in this work as was done in Kähm and Vassiliadis (2018). The differences in analysis with respect to the work in Kähm and Vassiliadis (2018) are obtained because in this work the most conservative gradient coefficients are used, and not the average values. Furthermore, slight differences arise due to the complication that it is very difficult to find the exact point where stability is lost.

The accordance of stability criterion $\mathcal{K}$ for the description of thermal stability in batch processes with the actual loss of thermal stability is analysed in detail in following section.

\subsection{Case studies for criterion $\mathcal{K}$}

As was done for the divergence criterion in Section 3.2.2, the stability criterion profiles are shown for systems which go from stable to unstable operation. It is important to note that a potential stability measure has to be conservative in the sense that instability is overpredicted. On the other hand it must not be too conservative, otherwise such a criterion cannot be used for process optimisation. Hence, the cross-over from the negative to positive value of $\mathcal{K}$ is of interest in the following graphs. The temperature profiles for processes $\mathrm{P}_{1}-\mathrm{P}_{20}$ are given in Figures $3-9$. The respective stability criterion profiles are given in Figures $18-21$. 


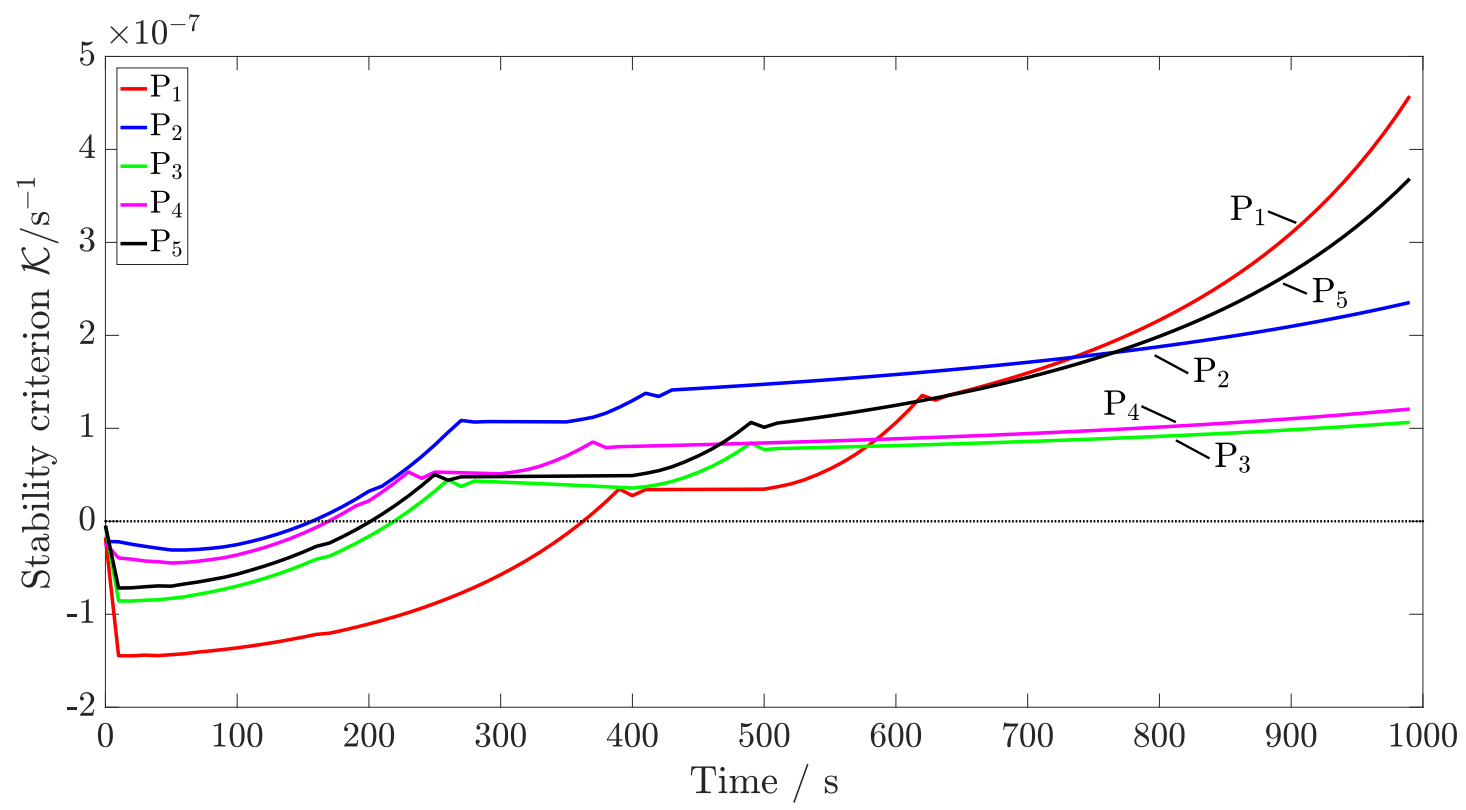

Figure 18: Stability criterion profiles for processes $\mathrm{P}_{1}-\mathrm{P}_{5}$.

${ }_{519}$ For processes $\mathrm{P}_{1}-\mathrm{P}_{5}$ the profiles for stability criterion $\mathcal{K}$ follow a similar trajectory to the temperature profile in Figure 2. Unlike the divergence criterion, there is a sign change before loss of stability: as the system becomes more unstable the value of $\mathcal{K}$ increases until it becomes positive. Once the value of $\mathcal{K}>0$, the system is predicted to be unstable. It can be seen from Figures 18 and 3 that instability is predicted before a thermal runaway occurs.

The stability criterion profiles for processes $\mathrm{P}_{6}-\mathrm{P}_{10}$ with respect to the temperature profiles in Figure 4 are shown in Figure 19. 


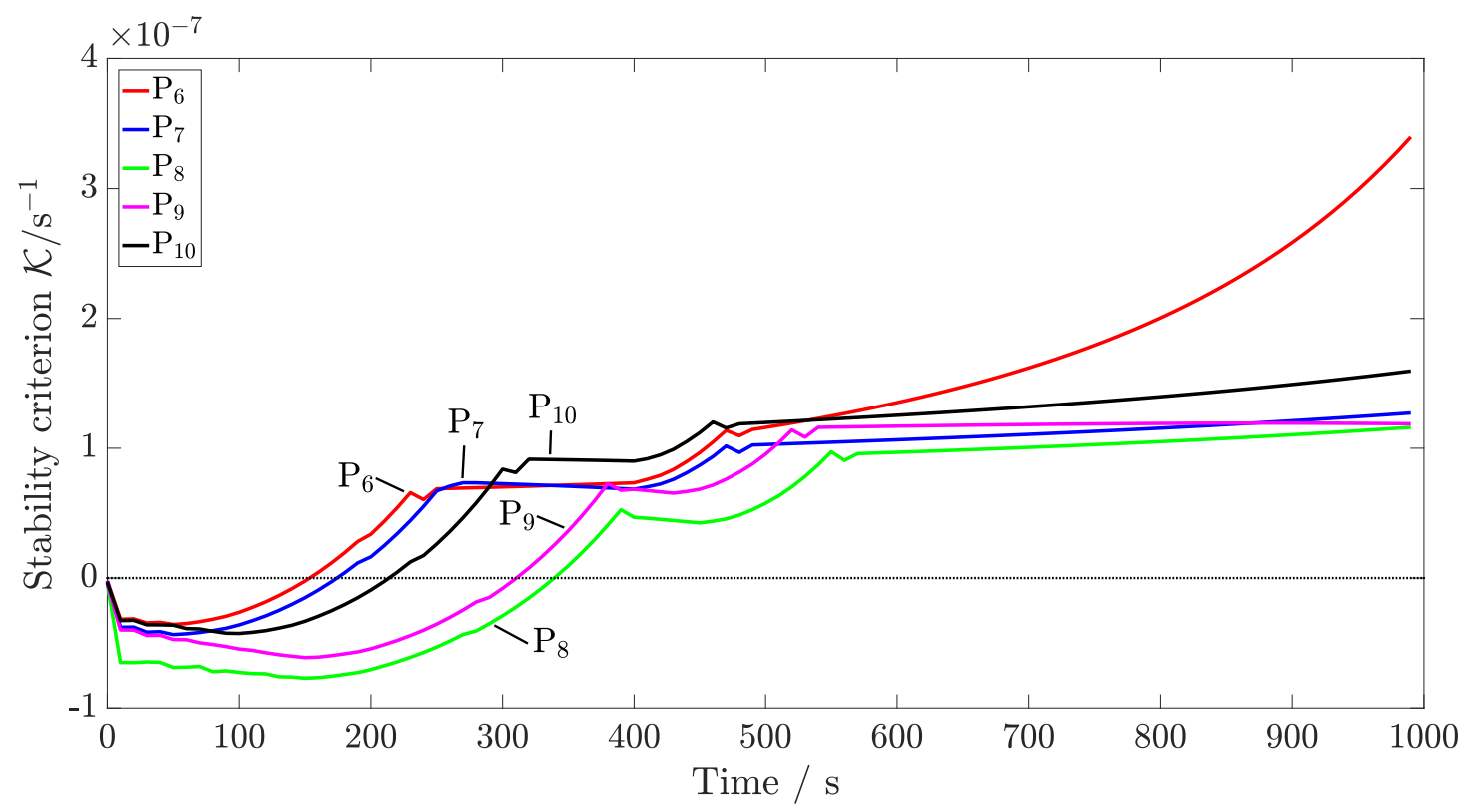

Figure 19: Stability criterion profiles for processes $\mathrm{P}_{6}-\mathrm{P}_{10}$.

${ }_{526}$ The points at which instability is predicted by criterion $\mathcal{K}$, i.e. the points where $\mathcal{K}$ ${ }_{527}$ becomes positive, correspond well with the actual loss of stability given by the temperature profiles given in Figure 4: as the temperature increases rapidly, the profiles for $\mathcal{K}$ increase in the same manner.

The stability criterion profiles for processes $\mathrm{P}_{11}-\mathrm{P}_{15}$ with respect to the temperature profiles in Figure 6 are shown in Figure 20.

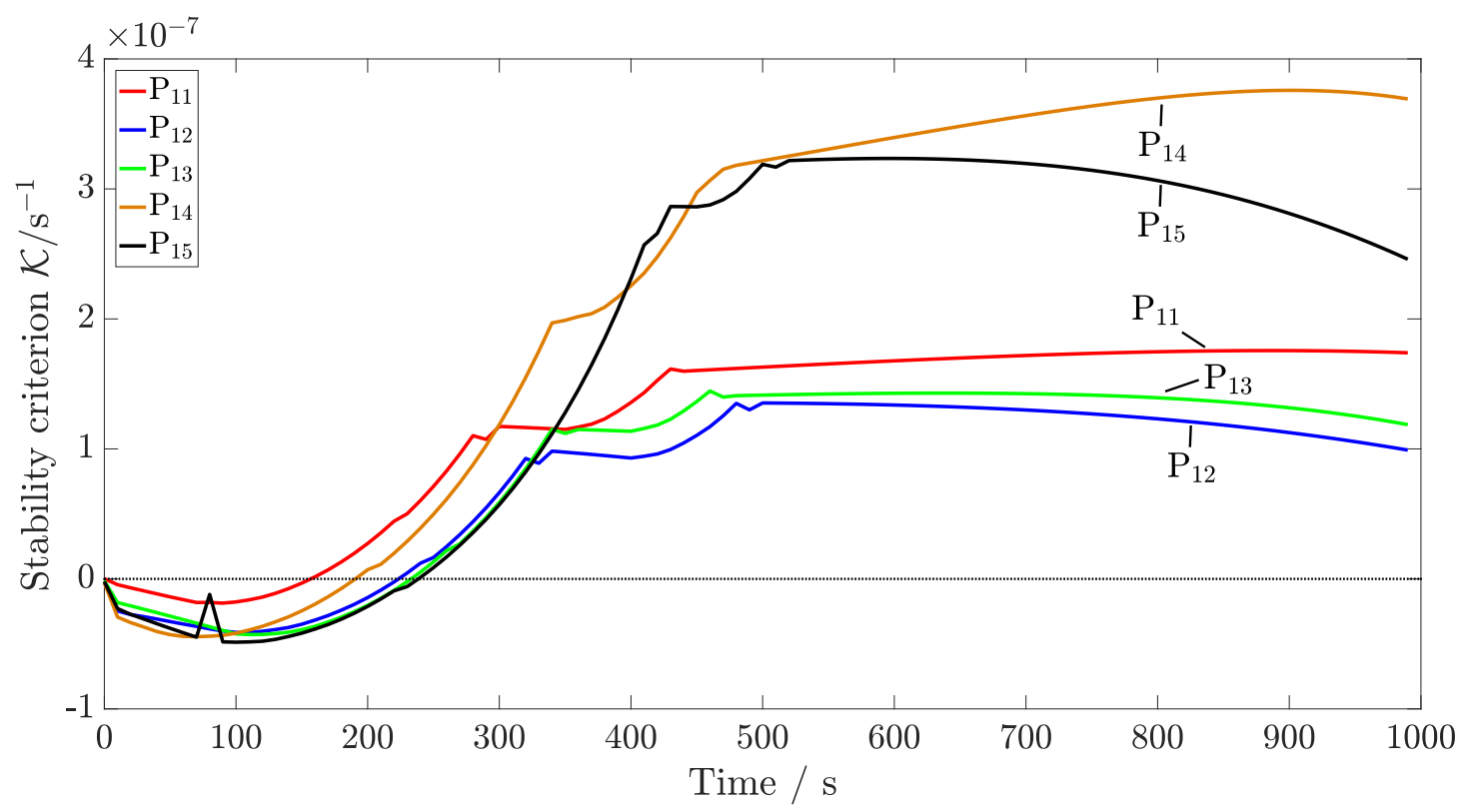

Figure 20: Stability criterion profiles for processes $\mathrm{P}_{11}-\mathrm{P}_{15}$. 
As was the case for processes $\mathrm{P}_{1}-\mathrm{P}_{10}$, instability predicted well by criterion $\mathcal{K}$ when compared to the temperature profiles in Figure 6. As was observed for the divergence in Figure 7 , the fast reaction leading to a reduction in reactants $\mathrm{A}$ and $\mathrm{B}$ leads to a reduction in the value for $\mathcal{K}$.

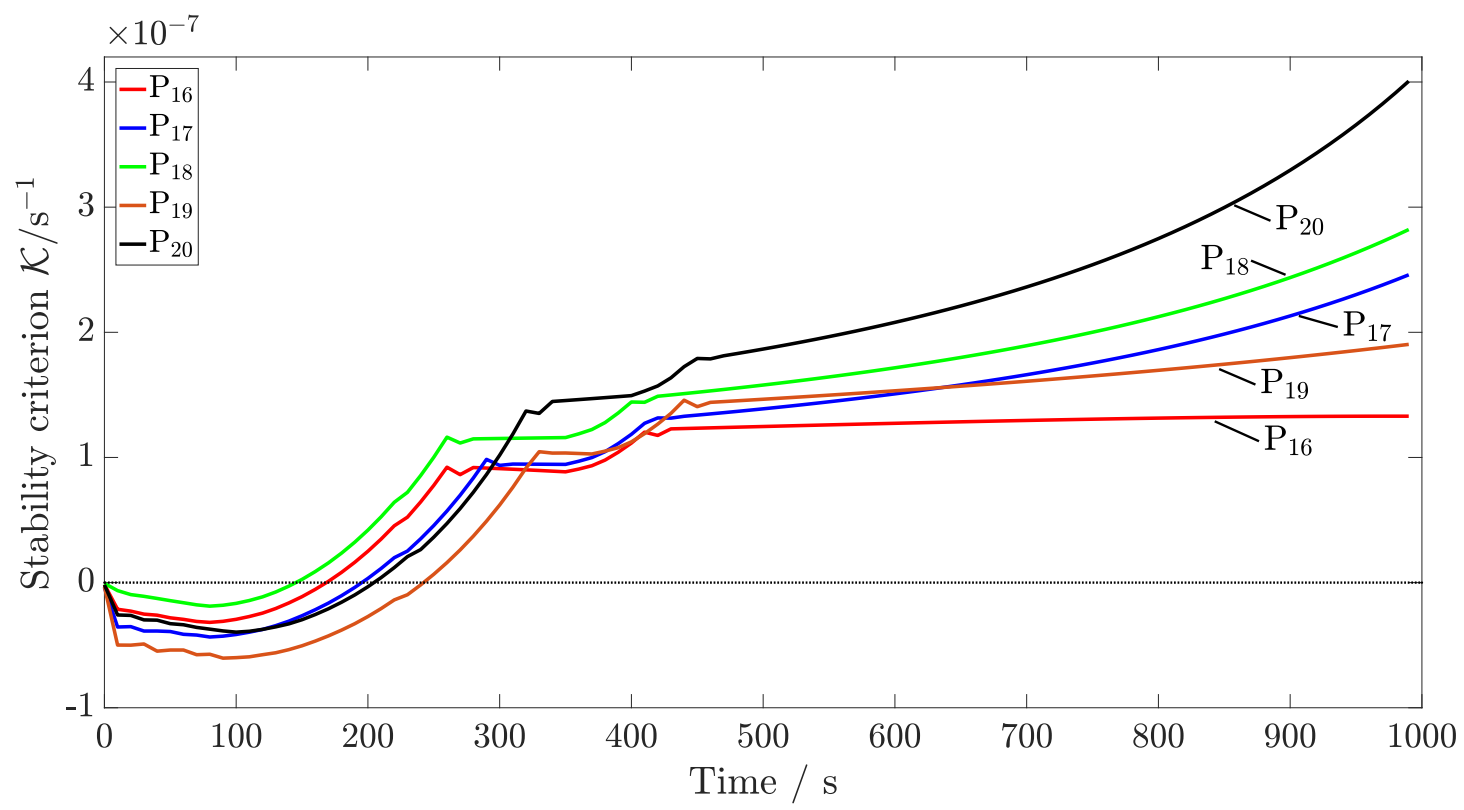

Figure 21: Stability criterion profiles for processes $\mathrm{P}_{16}-\mathrm{P}_{20}$.

For every process the sign change of criterion $\mathcal{K}$ occurs before loss of stability, as can be seen in Figures 18, 19, 20 and 21. The instability is predicted approximately $5 \mathrm{~K}$ before the system actually leads to a thermal runaway. Hence a more conservative prediction of the system stability is obtained. This is a positive feature, as a more conservative stability measure ensures the system stays within a stable operating region. Stability criterion $\mathcal{K}$ constitutes a much less conservative stability measure than the divergence criterion, hence allowing process intensification to be carried out. The slight conservative nature of criterion $\mathcal{K}$ gives a margin of error in case of parameter uncertainty or process disturbances, which could result in mistakenly classifying the nature of the system. A measure which were not to be conservative, if it were to exist, could result in an unstable system when implemented within MPC if slight process disturbances occur.

Hence the application of stability criterion $\mathcal{K}$ will give a control system which is able to predict system stability at the current point, without the need of further simulation and hence computational cost. Therefore the use of $\mathcal{K}$ with nonlinear MPC schemes will lead to significant reductions in reaction time without loss of stability.

In tank reactors with high turbulence due to the stirrer there is a near uniform distribution of temperature and concentration. The only change in properties occurs within a 
thin boundary layer which is negligible in comparison to the volume of the reacting mixture. The function for stability criterion $\mathcal{K}$, which is based on ideally mixed batch reactors, can be extended to non-ideally mixed reactors: if the stirrer does not result in ideal mixing, the properties and dimensionless variables used for stability criterion $\mathcal{K}$ have to be found as averages throughout the reactor volume.

\section{Intensification of batch processes with Model Predictive Control}

\subsection{Model Predictive Control applied to batch reactors}

Model Predictive Control (MPC) is an advanced control scheme, in which an Optimal Control Problem (OCP) is solved iteratively (Chuong La et al., 2017; Mayne, 2014). The analysis of stability of batch processes is incorporated into the classical MPC flow sheet, which is shown in Figure 22.

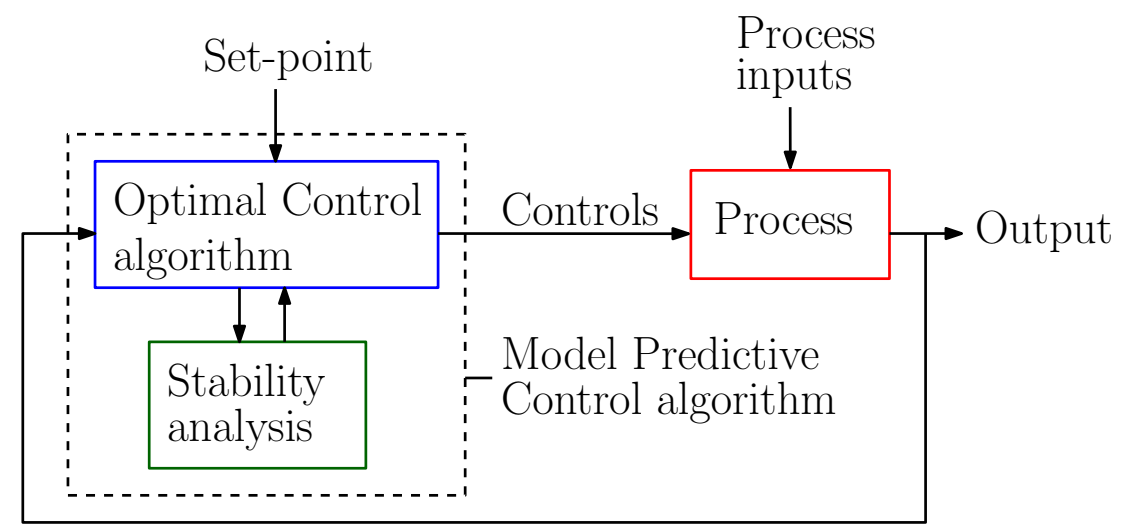

Figure 22: Model Predictive Control scheme with integrated stability analysis.

At every iteration process data is used to find the optimal control strategy, together with an estimate of the system stability for the evaluated control strategy. The stability is implemented as a constraint which, if not satisfied, leads to the optimisation algorithm re-evaluating the optimal control strategy. As is given in Figure 22, the inputs to the advanced control algorithm are process inputs which can include disturbances and a set-point. Depending on what the system is required to do, the set-point can be redefined.

In order to intensify batch processes, the set-point temperature can be set to the maximum allowable temperature of the system, as the stability constraint will restrict the system to increase in temperature too fast and enter an unstable regime. Furthermore there is the option to maximise yield of a certain chemical, which lets the control system decide on its own by how much the temperature can be increased. For certain reactions the highest possible reaction temperature, without causing thermal runaways, is the target (Rupp et al., 2013). 
The mathematical formulation for MPC at the $i^{\text {th }}$ step used in this work is given by (Charitopoulos and Dua, 2016; Rawlings and Mayne, 2015):

$$
\min _{q_{\mathrm{C}}} \Phi^{(i)}\left(x(t), q_{\mathrm{C}}(t)\right)
$$

where $\Phi^{(i)}$ is the objective function of the optimisation, and $x(t)$ are the state variables of the system described in Equations (2.1) - (2.7). This optimisation problem is subject to:

$$
\begin{array}{rlrl}
\Phi^{(i)} & = & & -\int_{f}^{t_{f}^{(i)}} X_{\mathrm{A}}(t) d t \\
0 & = & h\left(x(t), q_{\mathrm{C}}(t), t\right) \\
T_{\mathrm{R}} \leq & T_{\text {chem }} \\
0 \leq q_{\mathrm{C}}^{(i)} & \leq & q_{\mathrm{C}, \max } \\
\left|q_{\mathrm{C}}^{(i)}-q_{\mathrm{C}}^{(i-1)}\right| \leq & \delta q_{\mathrm{C}} \\
t_{0} \leq t \leq & t_{f}
\end{array}
$$

where $X_{\mathrm{A}}(t)$ is the conversion of reagent $\mathrm{A}$ and $h\left(x(t), q_{\mathrm{C}}(t), t\right)$ are the equations with respect to physical properties. The initial time and final time of the simulation at step $i$ are $t_{0}^{(i)}$ and $t_{f}^{(i)}$, respectively, and the chemical stability temperature is set to $T_{\text {chem }}=450 \mathrm{~K}$. This constraint is included in all following MPC schemes, as this represents a process constraint irrespective of the control system employed. The change in coolant flow rate between steps $i$ and $i-1, q_{\mathrm{C}}^{(i)}-q_{\mathrm{C}}^{(i-1)}$, is limited to at most equal to $\delta q_{\text {mathrmC }}$, which is set to $\delta q_{\mathrm{C}}=0.05 q_{\mathrm{C}, \max }$.

The following constraint is added to the set of equations for the optimisation:

$$
\mathcal{K}\left(t_{f}^{(i)}\right) \leq 0
$$

This constraint is included in order to keep the process in a stable region by satisfying criterion $\mathcal{K}$ at the final time of the respective optimisation, here $t_{f}^{(i)}$.

The batch system described by Equations $(5.1 a)$ - (5.1h) is solved using the SQP optimisation (Nocedal and Wright, 2006) algorithm within fmincon in MATLAB ${ }^{\mathrm{TM}}$. A sequential approach for the optimal control problem was implemented for the MPC framework.

A moving horizon approach is implemented, for which the optimal control action is found given data from the past and a process model. Depending on the control and prediction horizons $t_{c}$ and $t_{p}$, respectively, the performance of the MPC scheme can be tuned. The larger the prediction and control horizons, the higher the computational time per iteration. A more detailed discussion of this approach is given in Christofides et al. (2011), Haber et al. 
(2011) and Kähm and Vassiliadis (2018).

Only the first control step found by the optimisation algorithm is implemented according to the moving horizon approach. After this step is completed, the next iteration of the MPC scheme commences.

\subsection{Analysis of $M P C$ schemes and process intensification}

Using stability criterion $\mathcal{K}$ the process under consideration can be intensified. This is done by increasing the temperature of the process, whilst ensuring that criterion $\mathcal{K}$ is below zero, as was outlined above. Six processes with three different MPC algorithms are going to be considered in detail. The advantages of using stability criterion $\mathcal{K}$ are demonstrated in terms of computational time and process efficiency.

\subsubsection{MPC algorithms implemented}

MPC is used to keep each process under control. For each process, three MPC schemes are considered:

1. MPC with stability criterion $\mathcal{K}$

2. MPC scheme with constant set point temperature

3. MPC scheme with extended prediction horizon

The first scheme is the novel scheme which was outlined in the section above. This MPC scheme uses a control horizon of $t_{c}=50 \mathrm{~s}$ with five control increments, each with length of $10 \mathrm{~s}$, and no extended prediction horizon.

The second scheme is often found in industry: rather than increasing the temperature set-point during a process, it is easier to keep the reaction temperature constant in order to ensure stability of operation. This MPC scheme uses a control horizon of $t_{c}=50 \mathrm{~s}$ with five control increments, each with length of $10 \mathrm{~s}$, and no extended prediction horizon.

The third scheme is an alternative to using stability criteria altogether: as the prediction horizon of the MPC formulation is extended, the optimisation algorithm should be able to find control inputs which keep the system close to the desired temperature set point and within the defined constraints.

These three schemes are compared with respect to reliability of control and computational cost. The control horizon for this scheme is set to $t_{c}=50 \mathrm{~s}$ with five control increments, each with length of $10 \mathrm{~s}$, and a prediction horizon of $t_{p}=1000 \mathrm{~s}$. During the prediction horizon a constant control input is used. The value of this control input is given by the last control value within the control horizon of the MPC algorithm. 


\subsubsection{Temperature profiles of analysed $M P C$ systems}

As sample case studies processes $\mathrm{P}_{3}, \mathrm{P}_{5}, \mathrm{P}_{7}, \mathrm{P}_{9}, \mathrm{P}_{11}$ and $\mathrm{P}_{13}$ are considered, the process parameters of which can be found in Table 1 . The initial temperature for processes $\mathrm{P}_{3}, \mathrm{P}_{5}$ and $\mathrm{P}_{7}$ is set to $400 \mathrm{~K}$, whereas for processes $\mathrm{P}_{9}, \mathrm{P}_{11}$ and $\mathrm{P}_{13}$ the initial temperature is set to $405 \mathrm{~K}$. The temperature profiles for each MPC scheme applied to all processes are shown in Figures $23-25$.

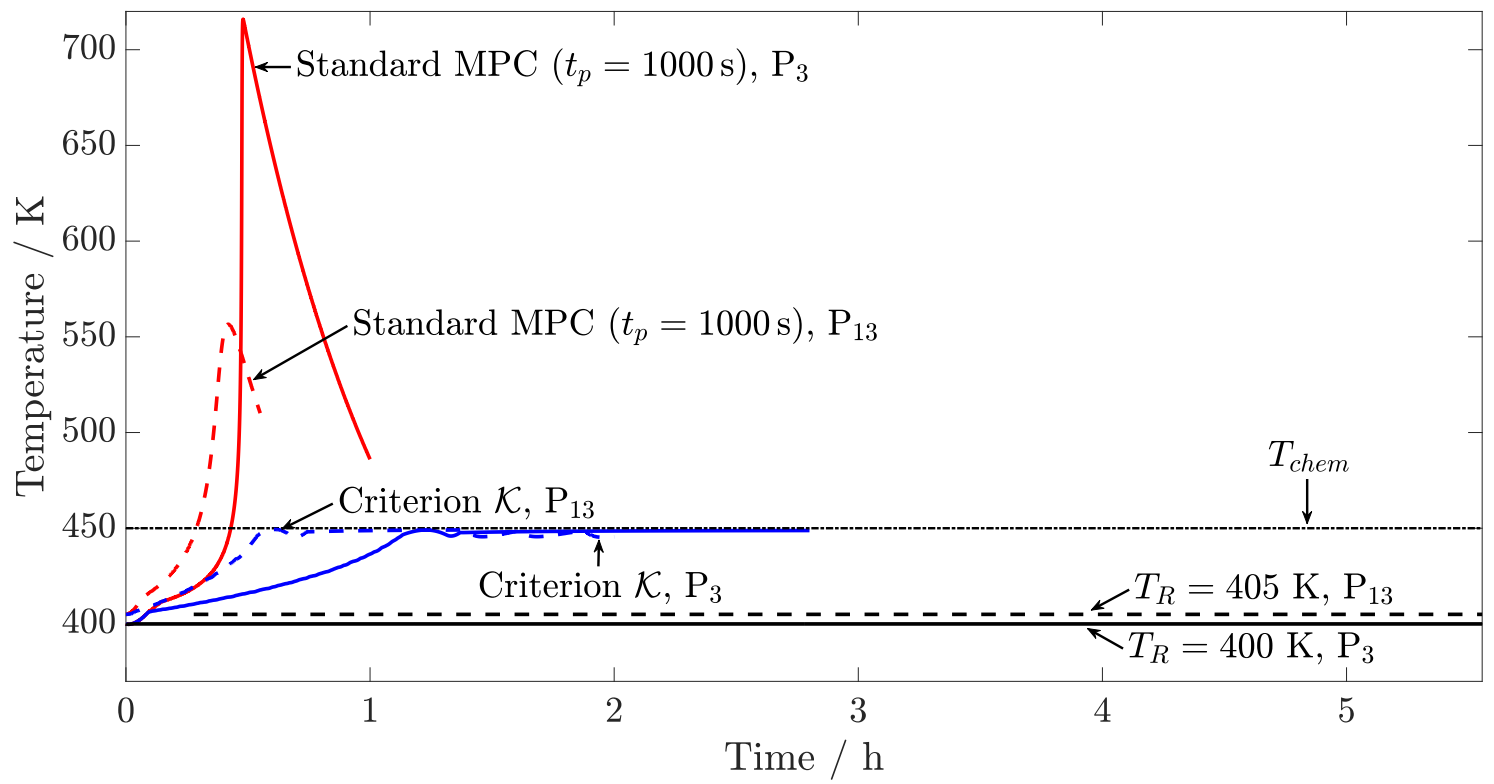

Figure 23: Temperature profiles of processes $\mathrm{P}_{3}$ and $\mathrm{P}_{13}$ for each MPC scheme.

(1)

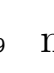

prctis

pre

$T_{\mathrm{R}}$

will be further illustrated by plots of the conversion for these reactions. 


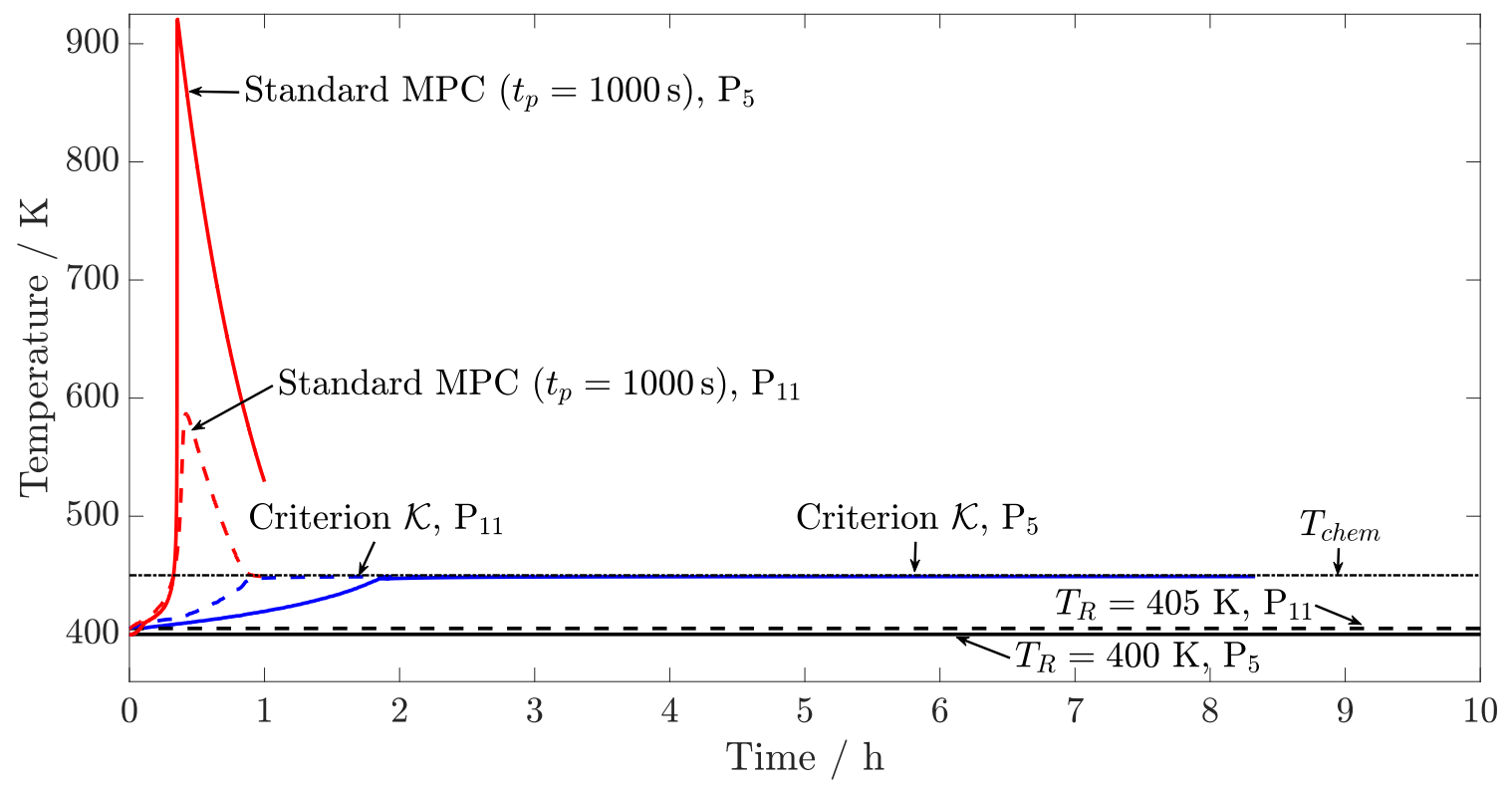

Figure 24: Temperature profiles of processes $\mathrm{P}_{5}$ and $\mathrm{P}_{11}$ for each MPC scheme.

644 For processes $\mathrm{P}_{5}$ and $\mathrm{P}_{11}$, shown in Figure 24 the same behaviour as for processes $\mathrm{P}_{3}$ and ${ }_{645} \mathrm{P}_{13}$ is observed. The MPC scheme with criterion $\mathcal{K}$ embedded gives stable operation while steadily increasing the reactor temperature. A constant temperature set-point with standard 


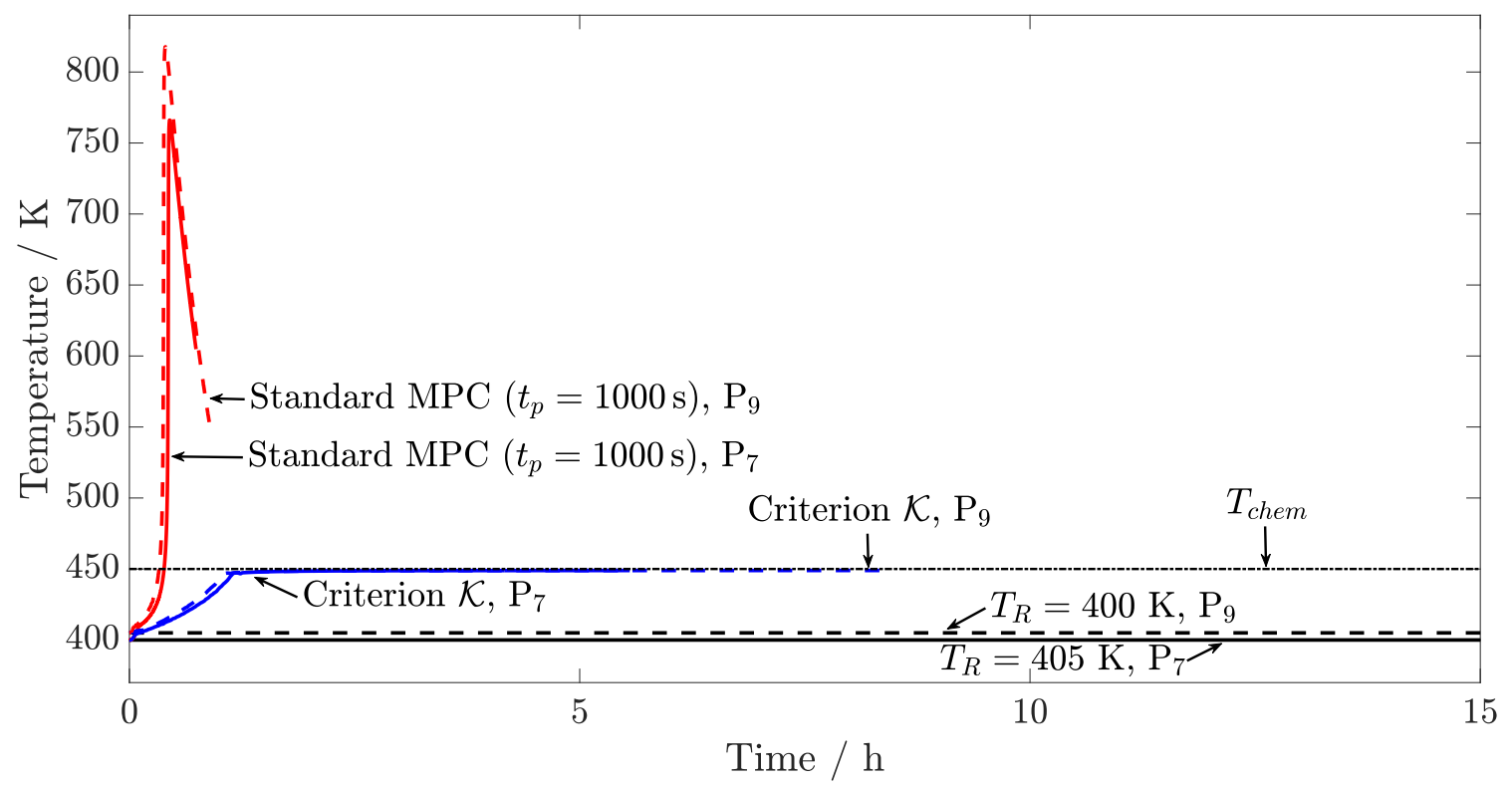

Figure 25: Temperature profiles of processes $\mathrm{P}_{7}$ and $\mathrm{P}_{9}$ for each MPC scheme.

For the last two processes considered in this work, processes $\mathrm{P}_{7}$ and $\mathrm{P}_{9}$, the same result is obtained as for the 4 previous case studies. As can be seen in Figure 25 stable operation is obtained for the first two MPC schemes using criterion $\mathcal{K}$ and a constant set point temperature. For the scheme using criterion $\mathcal{K}$ a controlled increase in the reactor temperature is observed until the maximum allowable temperature of $T_{\text {chem }}=450 \mathrm{~K}$ is reached. The temperature is kept below $T_{\text {chem }}$ at all times. The MPC scheme using a constant temperature set-point, as expected, gives a controlled process at that temperature. The MPC scheme trying to keep the system under control by having an extended prediction horizon gives a clear thermal runaway, reaching maximum temperatures of $T_{\mathrm{R}}=760 \mathrm{~K}$ for process $\mathrm{P}_{7}$ and $T_{\mathrm{R}}=820 \mathrm{~K}$ for process $\mathrm{P}_{9}$.

To show further the improved control obtained when embedding criterion $\mathcal{K}$ within an MPC scheme, additional simulations of processes $\mathrm{P}_{3}, \mathrm{P}_{5}, \mathrm{P}_{7}, \mathrm{P}_{9}, \mathrm{P}_{11}$ and $\mathrm{P}_{13}$ are shown. For these processes a standard MPC scheme, as in scheme 3 above, is employed with an extended prediction and control horizon. The time length for each control step is increased from $10 \mathrm{~s}$ to $100 \mathrm{~s}$ with only 3 control steps used instead of 5 , therefore increasing the control horizon from $50 \mathrm{~s}$ to $300 \mathrm{~s}$, and the prediction horizon is increased from $1000 \mathrm{~s}$ to $3000 \mathrm{~s}$. The resulting temperature profiles for these processes are shown in Figure 26. 


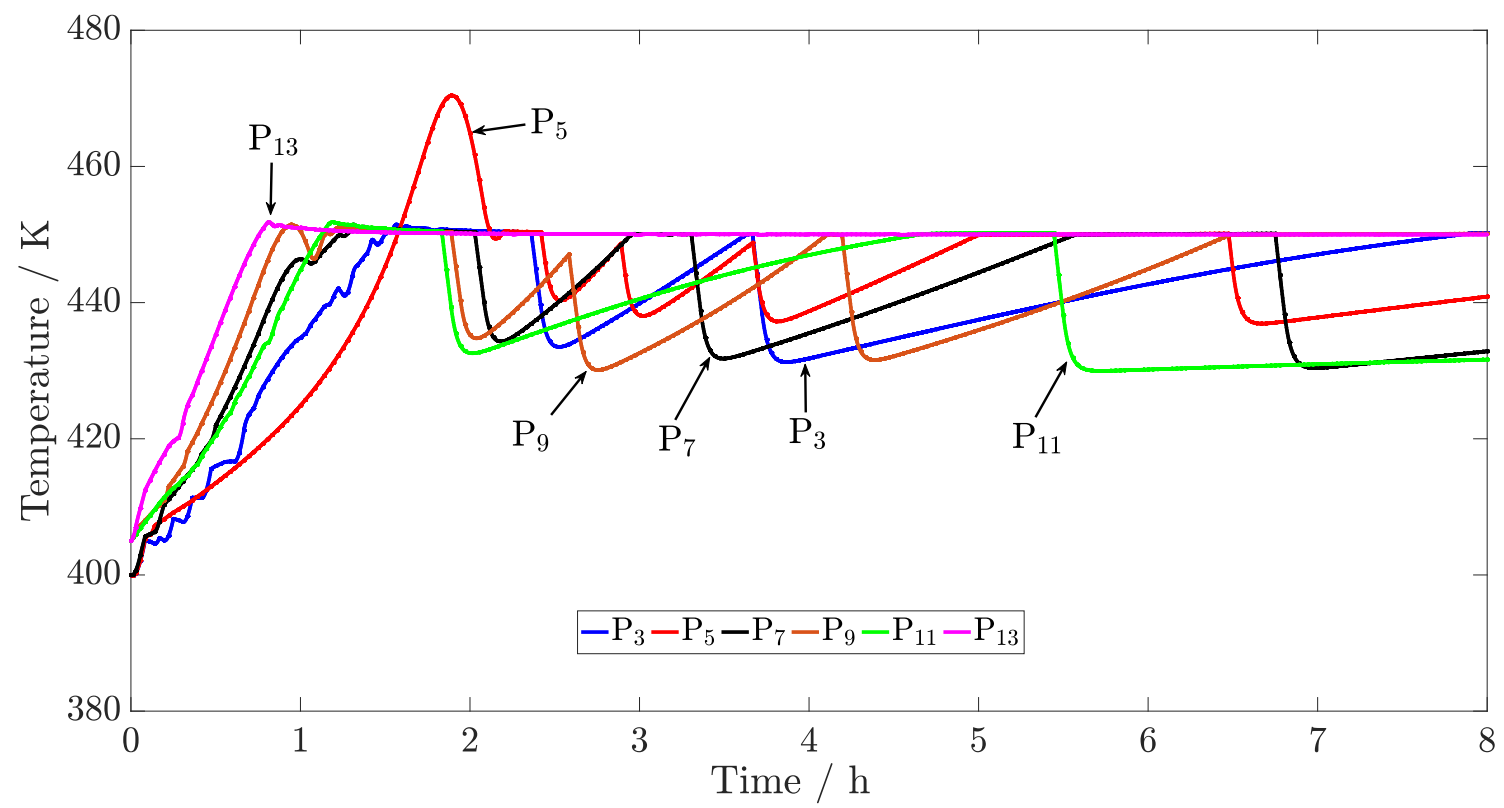

Figure 26: Temperature profiles for processes $\mathrm{P}_{3}, \mathrm{P}_{5}, \mathrm{P}_{7}, \mathrm{P}_{9}, \mathrm{P}_{11}$ and $\mathrm{P}_{13}$ when controlled by the modified MPC scheme with a control horizon of $300 \mathrm{~s}$ and a prediction horizon of $3000 \mathrm{~s}$.

In Figure 26 stable control is achieved for these processes, but the temperature profiles fluctuate more than the ones obtained with MPC scheme 1 embedding criterion $\mathcal{K}$ as an additional constraint. In order to achieve this stabilising control, the constraint on the rate of change of cooling given in Equation (5.1f) had to be relaxed from $\delta q_{C}=0.05 q_{\mathrm{C}, \max }$ to $\delta q_{C}=0.8 q_{\mathrm{C}, \max }$. This means that the cooling valve will be subject to larger sudden changes in position which can lead to a destabilised system. As discussed in Stephanopoulos (1984), such sudden variations in the control valve are not beneficial for the stability of systems.

\subsubsection{Analysis of computational time}

The first point of concern for this analysis is the computational cost required for each control scheme. This is of importance since these control schemes have to be implemented in an industrial setting. The lower the computational time for each iteration, the more likely is a successful implementation for online control schemes. In Table 12 the computational time for each control scheme and process are given. 
Table 12: Computational cost for each control scheme applied to processes $\mathrm{P}_{3}, \mathrm{P}_{5}, \mathrm{P}_{7}, \mathrm{P}_{9}, \mathrm{P}_{11}$ and $\mathrm{P}_{13}$. For the standard MPC scheme with an extended prediction horizon only the iterations before loss of stability are taken into account.

\begin{tabular}{lcccccc}
\hline \multirow{2}{*}{ MPC scheme } & \multicolumn{7}{c}{ Computational time / CPU s } \\
\hline With stability constraint $\mathcal{K}$ & 1.8 & 1.4 & 1.8 & 1.2 & 1.2 & 1.4 \\
Constant set point temperature & 0.70 & 0.50 & 0.60 & 0.50 & 0.50 & 1.0 \\
Standard MPC with extended horizon & 2.7 & 3.5 & 4.7 & 3.6 & 2.2 & 1.6 \\
Standard MPC with control horizon of & 1.3 & 1.1 & 1.4 & 1.3 & 1.4 & 2.0 \\
300 s and prediction horizon of 3000 s & & & & & & \\
\hline
\end{tabular}

As can be seen the constant set-point temperature MPC scheme results in the lowest computational cost. This is expected, since no additional constraints are added, therefore making the optimisation problem easier to solve. The MPC scheme using criterion $\mathcal{K}$ yields a lower computational cost than the MPC scheme with an extended prediction horizon. This is encouraging, as the system obtained by using criterion $\mathcal{K}$ also yields a more stable system. Hence the use of stability criterion $\mathcal{K}$ results in a faster and more reliable control scheme than conventional nonlinear MPC schemes.

The difference in computational time between the MPC scheme with constant set point temperature and MPC with stability criterion $\mathcal{K}$ is due to the interaction between the constraints and the optimisation algorithm. The actual time required to evaluate stability criterion $\mathcal{K}$ is less than $0.1 \mathrm{~s}$.

In order to yield a stable process with standard MPC schemes, that do not include any stability criteria, with increasing system temperature, an even longer prediction horizon will be required. This in turn can result in higher computational time which becomes a limiting factor for industrial applications.

To circumvent this issue, the number of control steps can be reduced, whilst increasing the time frame of each one. In this manner an MPC scheme as scheme 3 is considered as was shown in Figure 26. The computational cost for these case studies are shown in Table 12. It can be seen that the computational time can be decreased drastically whilst obtaining stable control as seen in Figure 26. This, on the other hand, comes at a compromise: The temperature profile of the system is not as smooth as for MPC scheme 1 including criterion $\mathcal{K}$, as the control increment allowable had to be increased to $80 \%$ to result in stable control. Therefore, using MPC scheme 1 results in more favourable operation.

Significant speed-up can be achieved by using $\mathrm{C}++$ or FORTRAN and faster computers. This does not change the fact that the MPC scheme using criterion $\mathcal{K}$ achieves the same goal 
in less computational time in a reliable manner.

\subsubsection{Process intensification}

The second concern of this analysis is the intensification of batch processes. For processes $\mathrm{P}_{3}, \mathrm{P}_{5}, \mathrm{P}_{7}, \mathrm{P}_{9}, \mathrm{P}_{11}$ and $\mathrm{P}_{13}$ given in Figures $23-25$ this is best illustrated by how long it takes each of them to reach the same conversion. The conversion profiles for processes $\mathrm{P}_{3}$ and $\mathrm{P}_{13}$ are shown in Figure 27, considering how long it takes to reach a target conversion of $75 \%$.

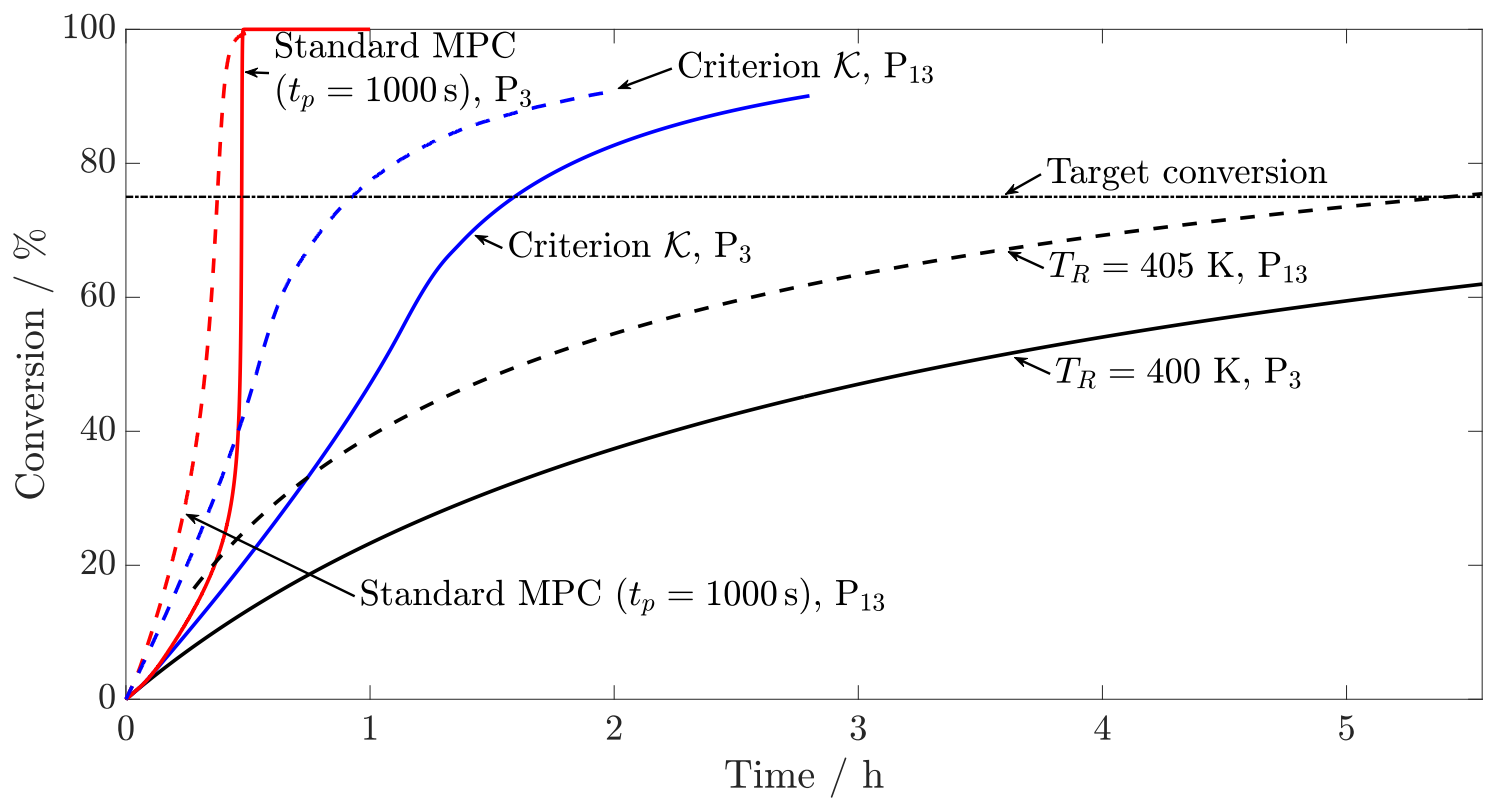

Figure 27: Conversion profiles of processes $\mathrm{P}_{3}$ and $\mathrm{P}_{13}$ for each MPC scheme.

From Figure 27 it can be seen clearly that the processes controlled by MPC with an extended prediction horizon yield thermal runaways, as the conversion reaches $100 \%$ after only $0.5 \mathrm{~h}$.

For process $\mathrm{P}_{3}$ the conversion for the MPC scheme with constant set-point temperature does not reach the target conversion of $75 \%$ even after $5.5 \mathrm{~h}$, whereas for process $\mathrm{P}_{13}$ the target conversion for this MPC scheme is just reached after $5.5 \mathrm{~h}$.

The MPC scheme with stability criterion $\mathcal{K}$ embedded achieves the conversion of $75 \%$ in $2.2 \mathrm{~h}$ for process $\mathrm{P}_{3}$ and $1.4 \mathrm{~h}$ for process $\mathrm{P}_{13}$, much faster than the constant temperature set-point system, as well as stable operation throughout the process.

The conversion profiles for processes $\mathrm{P}_{5}$ and $\mathrm{P}_{11}$ are shown in Figure 28. 


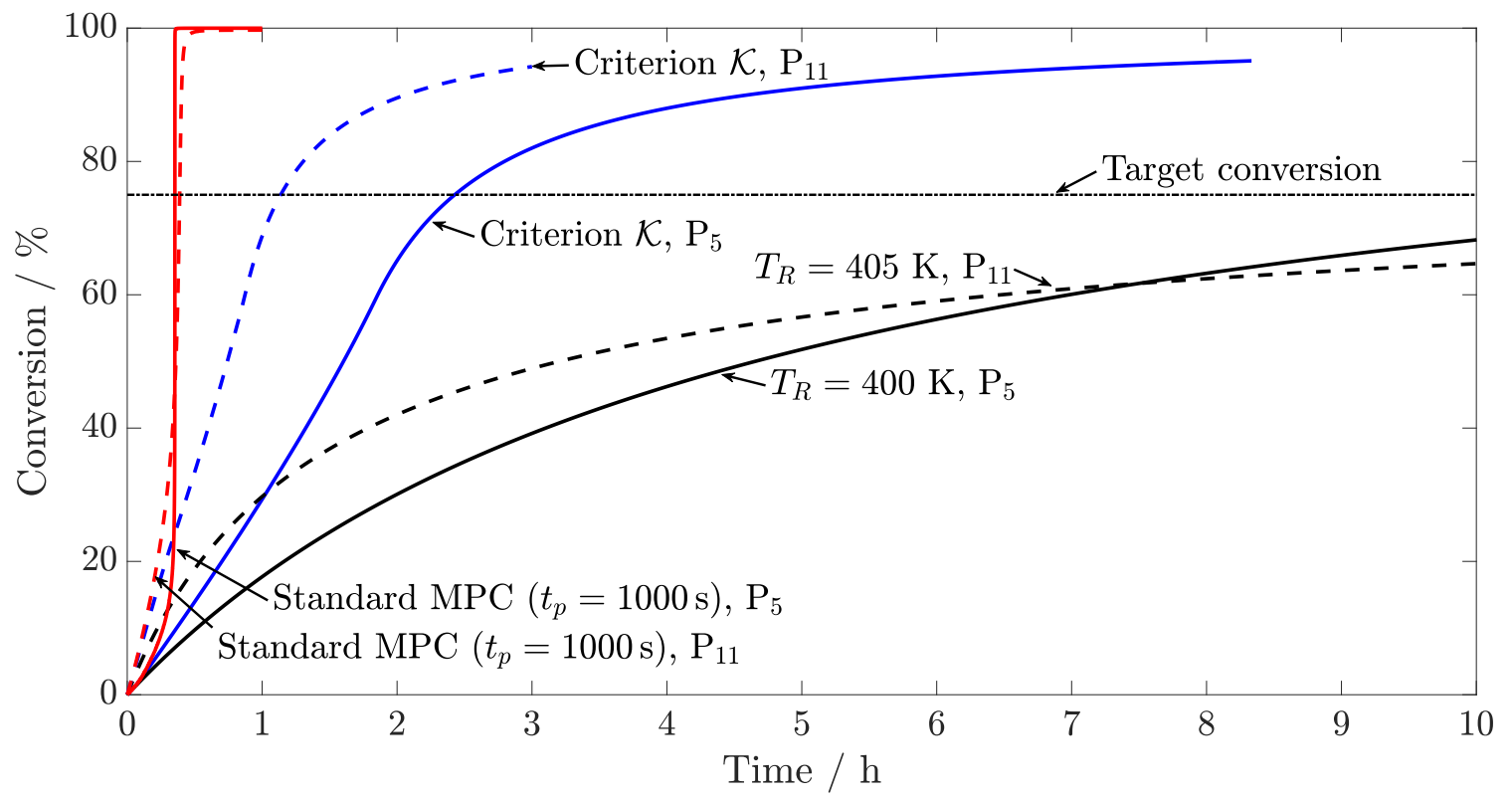

Figure 28: Conversion profiles of processes $\mathrm{P}_{5}$ and $\mathrm{P}_{11}$ for each MPC scheme.

The MPC scheme using a constant temperature set-point does not achieve the target conversion of $75 \%$ after $10 \mathrm{~h}$ for processes $\mathrm{P}_{5}$ and $\mathrm{P}_{11}$. Criterion $\mathcal{K}$ embedded within MPC results in processes that achieve the target conversion just after $1 \mathrm{~h}$ for process $\mathrm{P}_{11}$ and $2.2 \mathrm{~h}$ for process $\mathrm{P}_{5}$. With respect to the MPC scheme using constant temperature set-points this is a more than five-fold reduction in reaction time.

The MPC schemes using an extended prediction horizon, as can be seen in Figure 24, results in a thermal runaway. This can be seen by the sharp increase in conversion, reaching $100 \%$ after less than $0.5 \mathrm{~h}$ for both processes.

The conversion profiles for processes $\mathrm{P}_{7}$ and $\mathrm{P}_{9}$ are shown in Figure 29. 


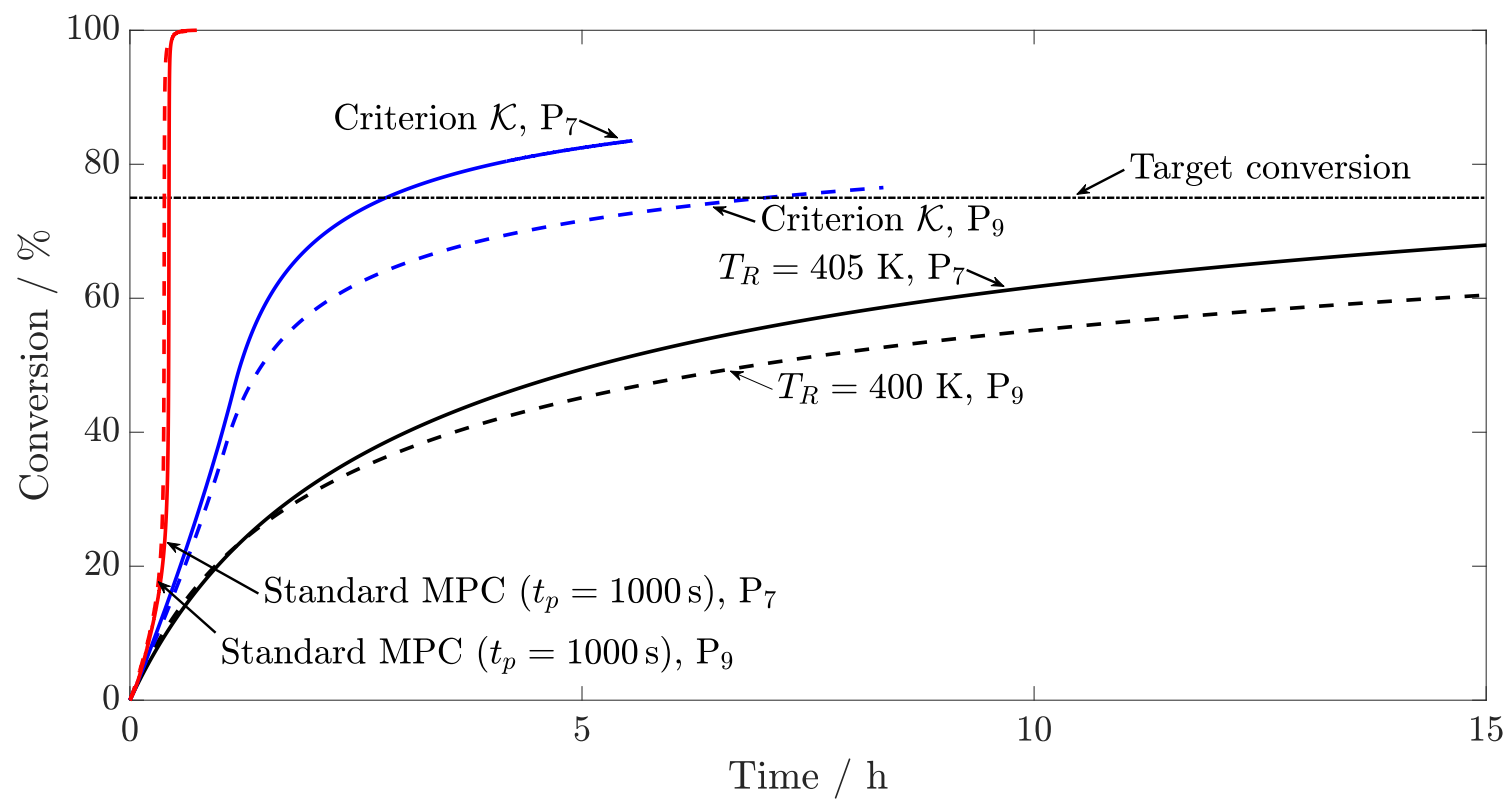

Figure 29: Conversion profiles of processes $\mathrm{P}_{7}$ and $\mathrm{P}_{9}$ for each MPC scheme.

The MPC schemes using criterion $\mathcal{K}$ result in stable processes reaching the target conversion after $2.5 \mathrm{~h}$ for process $\mathrm{P}_{7}$ and $7.5 \mathrm{~h}$ for process $\mathrm{P}_{9}$. This is longer than for the processes considered before: since the same concentrations of reactant A and B are initially present, as can be seen in Table 1 , as the reaction proceeds the rate of reaction decreases rapidly as both reactants are consumed.

Keeping a constant reactor temperature with the second MPC scheme hence gives an even longer reaction time, not reaching the target conversion after even $15 \mathrm{~h}$. Hence a reduction in reaction time of at least two-fold is achieved for processes $\mathrm{P}_{7}$ and $\mathrm{P}_{9}$.

Again, the third MPC scheme results in a thermal runaway, hence giving $100 \%$ conversion in a very short time span. The point where $100 \%$ conversion is reached coincides with the maximum temperature peaks seen in Figure 25.

The same behaviour as for processes $\mathrm{P}_{3}, \mathrm{P}_{5}, \mathrm{P}_{7}, \mathrm{P}_{9}, \mathrm{P}_{11}$ and $\mathrm{P}_{13}$ is found for all other process scenarios given in this work. Only these six processes are presented as a sample, as including results for all processes would add no further value to the analysis.

As can be seen from the case studies above, intensification of exothermic batch processes is achieved by integrating stability criterion $\mathcal{K}$ into standard MPC schemes as a nonlinear constraint. Not only is the reaction time reduced while keeping the process in a stable region, but the computational effort is also reduced in comparison to standard nonlinear MPC schemes with extended prediction horizons. To achieve the same stable operation, without any stability criterion, as was achieved by embedding criterion $\mathcal{K}$, even longer prediction and control horizons would be necessary - resulting in even higher computational overheads. 


\section{Conclusions and further work}

A more complex reaction scheme to the one found in Kähm and Vassiliadis (2018) is introduced for exothermic batch processes. Stability criterion $\mathcal{K}$ is derived and used to quantify the stability of these systems. It is found that modifications are required to make this criterion work for more complex reaction schemes. Furthermore, it is found that these modifications lead to the reliable prediction of instability for all reaction process scenarios considered in this work to illustrate the proposed methodology. For none of the simulated systems does stability criterion $\mathcal{K}$ not give a conservative estimate of the system behaviour. Similar gradient coefficients as for the simple reaction scheme found in Kähm and Vassiliadis (2018) are used. The results show that this scheme can be extended to other batch systems with complex reaction schemes.

Nonlinear Model Predictive Control (MPC) is introduced and the underlying methods used are elaborated. The stability criterion $\mathcal{K}$ is embedded in the MPC scheme as a nonlinear constraint, rather than a penalty term within the objective. It is found that the implementation of criterion $\mathcal{K}$ leads to an intensification of the process, while keeping the process in a stable regime. This in turn leads to decreased reaction times with improved safety, hence making it very useful for industry.

This improved efficiency is obtained due to the ability to increase the temperature of the reactor, while keeping the process under control. Furthermore it is found that the implementation of criterion $\mathcal{K}$ gives lower computational cost per MPC iteration with regards to standard nonlinear MPC schemes with extended prediction horizons. This means that the control scheme presented outperforms current MPC schemes in terms of stability, process efficiency and computational cost.

The contribution of this work is the extension and validation of a new stability criterion which is suitable for nonlinear non-steady state systems that can be incorporated into online control algorithms. Loss of stability has detrimental effects, resulting in industrial accidents and leading to economic loss. It is demonstrated that the novel methodology enhances safety and performance of processes that can become unstable.

The original divergence criterion uses first order derivatives, whereas the new stability criterion uses second order derivatives, hence making criterion $\mathcal{K}$ computationally more expensive. For batch processes it is very important to note that the original divergence criterion was proven to be too conservative to be useful for process intensification. The additional computational cost to calculate criterion $\mathcal{K}$ is therefore justified in order to improve the efficiency of the underlying processes.

Future work will focus on implementing more advanced MPC schemes to speed up the time required for each iteration. The computational cost, as well as accuracy, of using the 
divergence criterion and stability criterion $\mathcal{K}$ will hence be further analysed in future work when considering larger reaction systems. Reaction networks with several reactions in series and parallel will be considered also for the extension of this work. In order to predict the stability of such systems a suitable form of criterion $\mathcal{K}$ and the correction function $\mathcal{E}$ have to be found.

To improve further the MPC algorithm, sensitivity or adjoint equations could be incorporated in the optimisation step within the MPC algorithm, hence reducing the risk of numerical instabilities caused by numerical differentiation, which can occur from a finite differences approach as currently employed in this work.

The reliability of stability criterion $\mathcal{K}$ due to model-plant-mismatch have to be considered for future case studies. Ensuring a robust stability criterion for online applications is of major importance for a potential industrial application, hence requiring a detailed analysis in future work.

\section{Acknowledgments}

We thank the Engineering and Physical Sciences Research Council (EPSRC) and the Department of Chemical Engineering and Biotechnology, University of Cambridge, for funding the EPSRC PhD studentship for this project.

\section{References}

Anagnost, J. J., Desoer, C. A., 1991. An elementary proof of the Routh-Hurwitz stability criterion. Circuits Systems Signal Process 10, 101-114.

Arnold, V., 1973. Ordinary differential equations. MA: MIT Press, Cambridge, Ch. 3, pp. $95-208$.

Bohne, D., Fischer, S., Obermeier, E., May 2010. Thermal Conductivity, Density, Viscosity, and Prandtl-numbers of ethylene glycol-water mixtures. Berichte der Bundesgesellschaft für physikalische Chemie 88 (8), 739-742.

Bosch, J., Strozzi, F., Zbilut, J., Zaldívar, J. M., 2004. On-line runaway detection in isoperibolic batch and semibatch reactors using the divergence criterion. Computers and Chemical Engineering 28 (4), 527-544.

Charitopoulos, V. M., Dua, V., 2016. Explicit model predictive control of hybrid systems and multiparametrix mixed integer polynomial programming 62, 3441-2460.

Christofides, P. D., Liu, J., Muñoz de la Peña, D., 2011. Networked and Distributed Predictive Control. Springer, London, Ch. 2, pp. 13-45. 
Chuong La, H., Potschka, A., Bock, H. G., 2017. Partial stability for nonlinear model predictive control. Automatica 78, 14-19.

Copelli, S., Torretta, V., Pasturenzi, C., Derudi, M., Cattaneo, C., Rota, R., 2014. On the divergence criterion for runaway detection: Application to complex controlled systems. Journal of Loss Prevention in the Process Industries 28, 92-100.

Crittenden, J. C., Trussell, R. R., Hand, D. W., Howe, K. J., Tchobanoglous, G., 2012. MWH's Water Treatment: Principles and Design, 3rd Edition. John Wiley \& Sons, Ch. Appendix C, pp. 1861-1862.

Davis, M., Davis, R., 2003. Fundamentals of Chemical Reaction Engineering. McGraw-Hill, Ch. 2, pp. 53-56.

DeHaan, D., Guay, M., 2010. Model Predictive Control. Sciyo, Ch. 2, pp. 26-58.

Dever, J., George, K., Hoffman, W., Soo, H., 2004. Kirk-Othmer Encyclopedia of Chemical Technology. John Wiley \& Sons, Ch. Ethylene Oxide, pp. 632-673.

Ellis, M., Durand, H., Christofides, P. D., 2014. A tutorial review of economic model predictive control methods. Journal of Process Control 24, 1156-1178.

Green, D. W., Perry, R. H., 2008. Perry's Chemical Engineers' Handbook, eighth Edition. The McGraw-Hill, Ch. 2.

Haber, R., Bars, R., Schmitz, U., 2011. Predictive Control in Process Engineering. WileyVCH Verlag GmbH \& Co. KGaA, Ch. 2, pp. 29-54.

Hirschfelder, J. O., Curtiss, C. F., Bird, R. B., 1955. Molecular theory of gases and liquids. American Institute of Chemical Engineers Journal 1 (2), 272.

Hosen, M. A., Hussain, M. A., Mjalli, F. S., 2011. Control of polystyrene batch reactors using neural network based model predictive control (NNMPC): An experimental investigation. Control Engineering Practice 19, 454-467.

Huang, R., Biegler, L. T., Harianth, E., 2012. Robust stability of economically oriented infinite horizon NMPC that include cyclic processes. Journal of Process Control 22, 51-59.

Hurwitz, A., 1895. Über die Bedingungen, unter welchen eine Gleichung nur Wurzeln mit negativen reellen Theilen besitzt. Mathematische Annalen 46 (2), 273-284.

Kähm, W., Vassiliadis, V. S., 2018. Thermal stability criterion integrated in model predictive control for batch reactors. Chemical Engineering Science 188, 192-207. 
Kalmuk, A., Tyushev, K., Granichin, O., Yuchi, M., 2017. Online Parameter Estimation for MPC Model Uncertainties Based on LSCR Approach. In: 2017 IEEE Conference on Control Technology and Applications.

Kufoalor, D. K. M., Imsland, L., Johansen, T. A., 2015. Efficient implementation of step response prediction models for embedded model predictive control. In: 2015 IFAC Nonlinear Model Predictive Control.

Mayne, D., Michalska, H., 1990. Receding horizon control of nonlinear systems. IEEE Trans. on Automatic Control AC-35, 814-824.

Mayne, D. Q., 2014. Model predictive control: Recent developments and future promise. Automatica 50, 2967-2986.

Melcher, A., 2003. Numerische berechnung der lyapunov-exponenten bei gewöhnlichen differentialgleichungen. Ph.D. thesis, Universität Karlsruhe, Fakultät für Mathematik.

Nagy, Z. K., Braatz, R. D., 2003. Robust Nonlinear Model Predictive Control of Batch Processes. American Institute of Chemical Engineers Journal 49 (7), 1776-1786.

Nocedal, J., Wright, S., 2006. Numerical Optimization. Springer, Ch. 18, pp. 526-572.

Rawlings, J., Mayne, D., 2015. Model Predictive Control: Theory and Design. Nob Hill Publishing, Ch. 1, pp. 1-60.

Rossi, F., Copelli, S., Colombo, A., Pirola, C., Manenti, F., 2015. Online model-based optimization and control for the combined optimal operation and runaway prediction and prevention in (fed-)batch systems. Chemical Engineering Science 138, 760-771.

Routh, E., 1877. A treatise on the stability of a given state of motion: Particularly steady motion. Macmillan.

Rupp, M., Ruback, W., Klemm, E., 2013. Octanol ethoxylation in microchannels. Chemical Engineering and Processing: Process Intensification 74, 19-26.

Semenov, N., 1940. Thermal theory of combustion and explosion. In: Progress of Physical Science (U.S.S.R). Vol. 23.

Shampine, L., Reichelt, M., Kierzenka, J., 1999. Solving Index-1 DAEs in MATLAB and Simulink. SIAM Review 41, 538-552. 
Simon, L. L., Nagy, Z. K., Hungerbuehler, K., 2008. Nonlinear model predictive control of an industrial batch reactor subject to swelling constraint. In: Proceedings of the 17th World Congress The International Federation of Automatic Control.

Sinnot, R., 2005. Chemical Engineering Design. Vol. 6. Elsevier Butterworth-Heinemann, Ch. 12, pp. 634-638.

Stephanopoulos, G., 1984. Chemical Process Control. PTR Prentice Hall, Ch. 14, pp. 258279.

Strozzi, F., Zaldívar, J., 1994. A general method for assessing the thermal stability of batch chemical reactors by sensitivity calculation based on Lyapunov Exponents. Chemical Engineering Science 49 (16), 2681-2688.

Strozzi, F., Zaldívar, J., 1999. On-line runaway detection in batch reactors using chaos theory techniques. American Institute of Chemical Engineers Journal 45 (11), 2429-2443.

Teja, A. S., 1983. Simple method for the calculation of heat capacities of liquid mixtures. Journal of Chemical Engineering Data 28, 83-85.

van der Kloet, P., Neerhoff, F., 2003. Dynamic Eigenvalues and Lyapunov Exponents for Nonlinear Circuits. In: Proceedings of the Nonlinear Dynamics of Electronic Systems. 